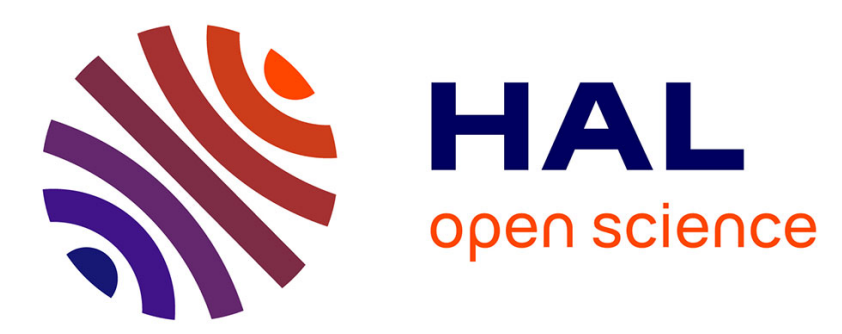

\title{
Mixed finite element methods: implementation with one unknown per element, local flux expressions, positivity, polygonal meshes, and relations to other methods
}

\author{
Martin Vohralík, Barbara Wohlmuth
}

\section{- To cite this version:}

Martin Vohralík, Barbara Wohlmuth. Mixed finite element methods: implementation with one unknown per element, local flux expressions, positivity, polygonal meshes, and relations to other methods. Mathematical Models and Methods in Applied Sciences, 2013, 23 (5), pp.803-838. 10.1142/S0218202512500613 . hal-00497394v3

\section{HAL Id: hal-00497394 \\ https://hal.science/hal-00497394v3}

Submitted on 16 Apr 2012

HAL is a multi-disciplinary open access archive for the deposit and dissemination of scientific research documents, whether they are published or not. The documents may come from teaching and research institutions in France or abroad, or from public or private research centers.
L'archive ouverte pluridisciplinaire HAL, est destinée au dépôt et à la diffusion de documents scientifiques de niveau recherche, publiés ou non, émanant des établissements d'enseignement et de recherche français ou étrangers, des laboratoires publics ou privés. 


\title{
Mixed finite element methods: implementation with one unknown per element, local flux expressions, positivity, polygonal meshes, and relations to other methods *
}

\author{
Martin Vohralík ${ }^{1}$ and Barbara Wohlmuth ${ }^{2}$
}

\author{
${ }^{1}$ UPMC Univ. Paris 06, UMR 7598, Laboratoire Jacques-Louis Lions, 75005, Paris, France \\ $\&$ \\ CNRS, UMR 7598, Laboratoire Jacques-Louis Lions, 75005, Paris, France \\ e-mail: vohralik@ann.jussieu.fr \\ ${ }^{2}$ Fakultät für Mathematik, Lehrstuhl für Numerische Mathematik \\ Boltzmannstrasse 3, 85748 Garching bei München, Germany \\ e-mail: wohlmuth@ma.tum.de
}

\begin{abstract}
In this paper, we study the mixed finite element method for linear diffusion problems. We focus on the lowest-order Raviart-Thomas case. For simplicial meshes, we propose several new approaches to reduce the original indefinite saddle point systems for the flux and potential unknowns to (positive definite) systems for one potential unknown per element. Our construction principle is closely related to that of the so-called multi-point flux-approximation method and leads to local flux expressions. We present a set of numerical examples illustrating the influence of the elimination process on the structure and on the condition number of the reduced matrix. We also discuss different versions of the discrete maximum principle in the lowest-order Raviart-Thomas method. Finally, we recall mixed finite element methods on general polygonal meshes and show that they are a special type of the mimetic finite difference, mixed finite volume, and hybrid finite volume family.
\end{abstract}

Key words: mixed finite element method, local static condensation, local flux expression, discrete maximum principle, polygonal mesh, locally conservative methods

\section{Introduction}

Let $\Omega \subset \mathbb{R}^{d}, d \geq 2$, be a polygonal (we use this term also in $\mathbb{R}^{d}, d \geq 3$ ) domain. We consider the pure diffusion model problem: find the potential $p: \Omega \rightarrow \mathbb{R}$ such that

$$
\begin{aligned}
-\nabla \cdot(\underline{\mathbf{S}} \nabla p)=g & \text { in } \Omega, \\
p=0 & \text { on } \partial \Omega,
\end{aligned}
$$

where $\underline{\mathbf{S}}: \Omega \rightarrow \mathbb{R}^{d \times d}$ is a symmetric, bounded, and uniformly positive definite diffusion tensor and $g: \Omega \rightarrow \mathbb{R}$ is a source term. Let $\mathcal{T}_{h}$ be a matching simplicial mesh of $\Omega$. For simplicity, we restrict

\footnotetext{
*The first author was supported by the GNR MoMaS project "Numerical Simulations and Mathematical Modeling of Underground Nuclear Waste Disposal", PACEN/CNRS, ANDRA, BRGM, CEA, EdF, IRSN, France.
} 
ourselves to the homogeneous Dirichlet boundary condition (1.1b) but our approach can be easily generalized to other boundary conditions. We also assume that $\underline{\mathbf{S}}$ and $g$ are piecewise constant on the mesh $\mathcal{T}_{h}$. We denote by $\mathbf{u}:=-\underline{\mathbf{S}} \nabla p$ the flux.

We are interested in the discretization of (1.1a)-(1.1b) by mixed finite elements (MFEs). This consists in finding $p_{h} \in \Phi_{h}$, an approximation to the potential $p$, and $\mathbf{u}_{h} \in \mathbf{V}_{h}$, an approximation to the flux $\mathbf{u}$, such that

$$
\begin{aligned}
\left(\underline{\mathbf{S}}^{-1} \mathbf{u}_{h}, \mathbf{v}_{h}\right)-\left(p_{h}, \nabla \cdot \mathbf{v}_{h}\right) & =0 & & \forall \mathbf{v}_{h} \in \mathbf{V}_{h}, \\
-\left(\nabla \cdot \mathbf{u}_{h}, \phi_{h}\right) & =-\left(g, \phi_{h}\right) & & \forall \phi_{h} \in \Phi_{h} .
\end{aligned}
$$

Here, we focus on the lowest-order Raviart-Thomas case, where the degrees of freedom of the potential space $\Phi_{h}$ are elementwise constants on $\mathcal{T}_{h}$, and the degrees of freedom of the flux space $\mathbf{V}_{h}$ represent the sidewise constant values of the normal component of the flux. The system (1.2a)$(1.2 \mathrm{~b})$ can be written in matrix form as

$$
\left(\begin{array}{ll}
\mathbb{A} & \mathbb{B}^{t} \\
\mathbb{B} & 0
\end{array}\right)\left(\begin{array}{l}
U \\
P
\end{array}\right)=\left(\begin{array}{c}
F \\
G
\end{array}\right)
$$

and is of indefinite, saddle point type. In our case, thanks to the homogeneous Dirichlet boundary condition (1.1b), $F=0$, but we prefer to write the general form (1.3) with $F$ not necessarily zero. The system (1.3) is well-posed; $\mathbb{A}$ is symmetric and positive definite and $\mathbb{B}$ has a full row rank. Thus, there exists a unique solution, on any simplicial mesh $\mathcal{T}_{h}$, without any restriction on the shape of the elements; the usual shape-regularity of the mesh is only necessary in convergence proofs. Recall also that the system (1.3) is well-posed in any space dimension. All these classical results can be found, e.g., in [16, 47, 54].

There has been a long-standing interest to reduce (1.3) to a system for the potentials $P$ only. The main motivations are to reduce the number of unknowns, to replace the saddle point system (1.3) by, if possible, a symmetric and positive definite one, and to relate the mixed finite element method to the finite difference and finite volume ones. A possible solution consists in using the first block equation of (1.3) to eliminate the unknowns $U$ through

$$
U=\mathbb{A}^{-1}\left(F-\mathbb{B}^{t} P\right) .
$$

Note that (1.4) represents a global flux expression, since $\mathbb{A}^{-1}$ as the inverse of a mass matrix is not sparse. Plugging (1.4) into the second block equation of (1.3), one can solve for $P$ the system

$$
\mathbb{B A}^{-1} \mathbb{B}^{t} P=\mathbb{B A}^{-1} F-G .
$$

The matrix $\mathbb{B A}^{-1} \mathbb{B}^{t}$ is symmetric and positive; however, it is full and not locally computable. In mixed Schur complement methods, the explicit construction of $\mathbb{B A}^{-1} \mathbb{B}^{t}$ is avoided by solving (1.4) in each step of an iterative process.

In the past, various approximate numerical quadratures have been used, see, e.g., $[48,4,11,8]$ (we give references in the order of publication) to reduce (1.3) into a system of the form

$$
\widetilde{\mathbb{S}} \widetilde{P}=\widetilde{H},
$$

with sparse, locally computable, and possibly symmetric and positive definite matrix $\widetilde{\mathbb{S}}$. In these approaches, however, because of the numerical quadratures, the new potentials $\widetilde{P}$ are, in general, different from the potentials $P$ in (1.3) and one cannot recover the exact potentials $P$.

One-unknown-per-element rewriting of (1.3) without any numerical quadrature in the form

$$
\mathbb{S} \bar{P}=H,
$$


where $\bar{P}$ is a new unknown from which $P$ can be locally recovered, has been achieved in two space dimensions in $[59,20,57]$ by exploiting an equivalence between lowest-order MFEs and finite volumes. A similar approach, with various extensions, has been taken in [43]. In [53], a system of the form (1.7) has been obtained with $\bar{P}=P$, i.e., directly for the original unknowns $P$, in two or three space dimensions. In these approaches, in contrast to (1.5), the matrix $\mathbb{S}$ is sparse and locally computable and, in contrast to (1.6), one obtains exactly the potentials $P$ of (1.3), by solving (1.7) and possibly performing a local postprocessing step. Intermediately, local flux expressions (enabling to recover the fluxes $U$ of (1.3) on sides of local patches from the potentials $P$ on elements of these patches) have been established in $[59,20,57,53]$. For alternative approaches to reduce the number of unknowns in $(1.3)$, we refer to $[6,51]$ and the references therein.

The first goal of the present paper is to unify the approaches of $[59,20,57]$ and of [53], to identify their common principles, and to show that they can be included in the general approach developed recently in [55]. We tackle this issue in Section 4, after summarizing the notation in Section 2 and recalling some basics in Section 3. In Section 3, we also recall the relation of the lowest-order Raviart-Thomas (RT0) mixed finite element (MFE) method to the Crouzeix-Raviart (CR) nonconforming finite element (NCFE) method and local flux expressions from the Lagrange multipliers.

The second goal of the present paper is to carry out a comparative numerical study of the different one-unknown-per-element RT0 MFE reformulations. In particular, we focus on symmetry, positive definiteness, sparsity, condition number, and performance of standard direct and iterative solvers in the different approaches in the presence of inhomogeneous and anisotropic diffusion tensors $\underline{\mathbf{S}}$. This numerical study is carried out in Section 5 .

The third goal of the present paper is to recall and state precisely the different variants of the discrete maximum principle valid in the RT0 MFE method. We do this in Section 6.

The fourth goal of the present paper is to recall that, in contrast to a widespread misleading belief, MFEs can be easily defined on general polygonal meshes. We present this result in Section 7 on quite general meshes and without loosing any accuracy with respect to (1.2a)-(1.2b). The presentation of this section is done for all order schemes. We simply introduce a matching simplicial submesh and use a local static condensation corresponding to the solution of local Dirichlet or Neumann problems.

The last goal of the present paper is to recall known and show new relations between $M F E$ methods and other discretization schemes, namely the two-point finite volume (FV) [29], mimetic finite difference (MFD) [17, 13], hybrid finite volume [31], mixed finite volume [26], multi-point flux-approximation (MPFA) [1, 3, 19], and related methods [28, 41, 14, 56, 42]. We in particular prove in Section 7 that the RTO MFE method on arbitrary polygonal meshes is a particular example of the MFD method. It seems that the only conceptual difference of the present MFE approach with these methods is that in MFEs, one has to construct a simplicial submesh and solve a local problem on each polygonal cell. For some other comparisons between these methods, we refer to [38] and the references therein. Finally, some conclusions are drawn in Section 8.

\section{Notation}

This section is devoted to collecting in one place the different notation used throughout the paper.

For a given domain $\omega \subset \mathbb{R}^{d}$, let $L^{2}(\omega)$ be the space of square-integrable functions over $\omega$ and $(\cdot, \cdot)_{\omega}$ the $L^{2}(\omega)$ inner product; we omit the index $\omega$ when $\omega=\Omega$. By $|\omega|$, we denote the Lebesgue measure of $\omega$ and by $|\sigma|$ the $(d-1)$-dimensional Lebesgue measure of a $(d-1)$-dimensional surface $\sigma$ in $\mathbb{R}^{d}$. The symbol $|S|$ also stands for the cardinality (the number of elements) of a set $S$. Finally, we denote by $\langle\cdot, \cdot\rangle_{\sigma}$ the $(d-1)$-dimensional $L^{2}(\sigma)$-inner product on $\sigma \subset \mathbb{R}^{d-1}$. 

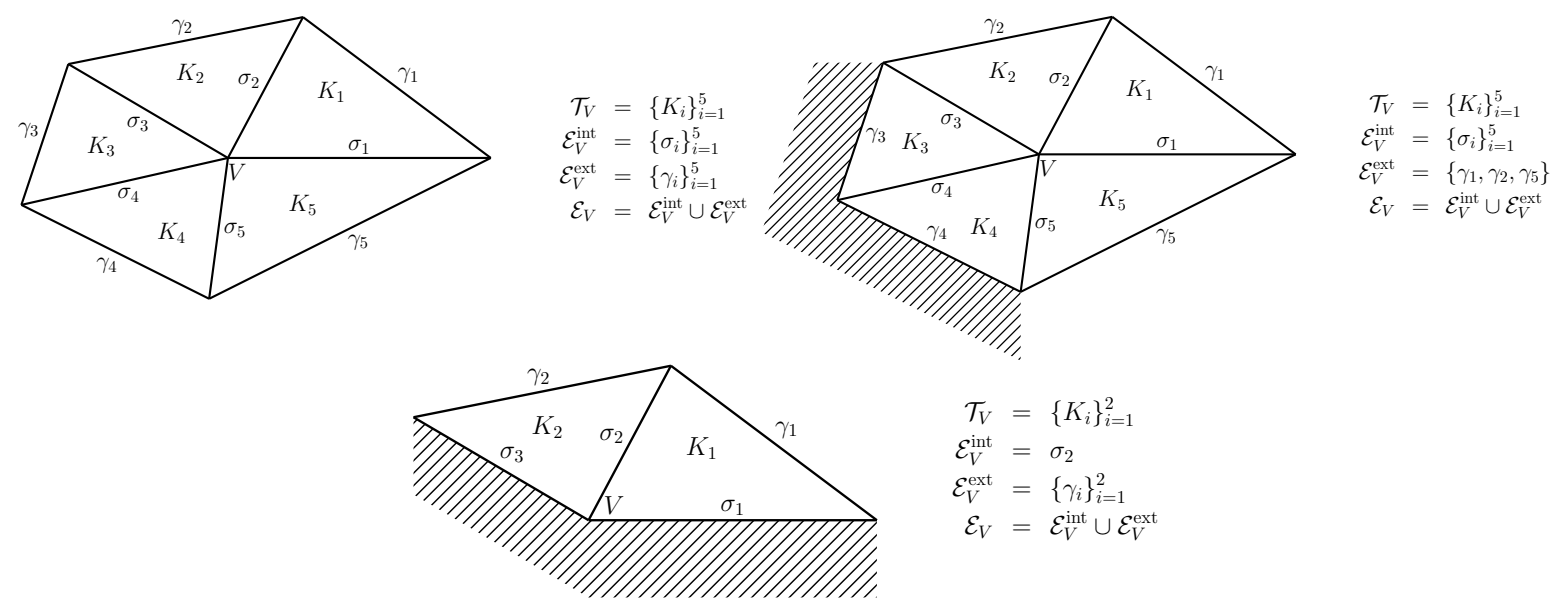

Figure 1: An example of a patch $\mathcal{T}_{V}$ around a vertex $V$ in the interior of $\Omega$ (top left), close to the boundary (top right), and on the boundary (bottom)

The elements of the mesh $\mathcal{T}_{h}$ are triangles in two space dimensions, tetrahedra in three space dimensions, and, in general, $d$-simplices. For simplicity, we exclude the pathological cases with just a couple of mesh elements. We suppose that $\mathcal{T}_{h}$ is matching, i.e., such that if $K, L \in \mathcal{T}_{h}, K \neq L$, then $K \cap L$ is either an empty set or a common vertex of $K$ and $L$ or a common $d^{\prime}$-face of the mesh $\mathcal{T}_{h}, 1 \leq d^{\prime} \leq d-1$. We denote by $\mathcal{E}_{h}$ the set of all sides of $\mathcal{T}_{h}$, i.e., the set of $(d-1)$-faces of the mesh $\mathcal{T}_{h}$. We divide $\mathcal{E}_{h}$ into the set $\mathcal{E}_{h}^{\text {int }}$ of interior sides and the set $\mathcal{E}_{h}^{\text {ext }}$ of boundary sides. Let $K \in \mathcal{T}_{h}$. By $\mathcal{E}_{K}$, we denote the set of all sides of $K$ and by $\mathcal{E}_{K}^{\text {int }}:=\mathcal{E}_{K} \cap \mathcal{E}_{h}^{\text {int }}$. For $K \in \mathcal{T}_{h}$, let $\mathbf{n}_{K}$ be the outward unit normal vector defined on the sides of $K$, and, for $\sigma \in \mathcal{E}_{h}$, let $\mathbf{n}_{\sigma}$ stand for the unit normal vector of $\sigma$ whose orientation is chosen arbitrarily but fixed for interior sides and coinciding with the exterior normal of $\Omega$ for boundary sides. We denote by $\mathcal{V}_{h}$ the set of vertices of $\mathcal{T}_{h}$. For a given vertex $V \in \mathcal{V}_{h}$, we shall denote by $\mathcal{T}_{V}$ the patch of the elements of $\mathcal{T}_{h}$ which share $V$, by $\mathcal{E}_{V}$ those sides of the elements in $\mathcal{T}_{V}$ contained in $\mathcal{E}_{h}^{\text {int }}$, and by $\mathcal{E}_{V}^{\text {int }}$ the sides in the interior of $\mathcal{T}_{V}$. We set $\mathcal{E}_{V}^{\text {ext }}:=\mathcal{E}_{V} \backslash \mathcal{E}_{V}^{\text {int }}$. Figure 1 gives various examples. We will also employ the notation $\mathcal{T}_{V}^{\text {ext }}$ for all elements from $\mathcal{T}_{V}$ having a side from the set $\mathcal{E}_{V}^{\text {ext }} ; \mathcal{T}_{V}^{\text {ext }}=\mathcal{T}_{V}$ apart from the second situation of Figure 1. We denote by $\mathcal{E}_{V, K}$ the sides of $K$ which have $V$ as vertex. For $K \in \mathcal{T}_{h}$, let $\mathbf{x}_{K}$ stand for the barycenter of $K$ and for $\sigma \in \mathcal{E}_{h}$, let $\mathbf{x}_{\sigma}$ stand for the barycenter of $\sigma$. We will also employ the notation $\mathcal{T}_{\sigma}$ for the patch of the elements of $\mathcal{T}_{h}$ which share the side $\sigma \in \mathcal{E}_{h}$. Finally, $\mathbb{I}$ denotes the identity matrix.

\section{The lowest-order Raviart-Thomas mixed finite element method}

We recall here some well-known properties of the RT0 MFE method. First, in Section 3.1, we define properly the spaces $\Phi_{h}$ and $\mathbf{V}_{h}$. Section 3.2 gives the hybridization and Section 3.3 recalls its relation to the Crouzeix-Raviart nonconforming finite element, as well as some other methods. In Section 3.4, we then present the local flux expressions from the Lagrange multipliers.

\subsection{Spaces $\Phi_{h}$ and $\mathbf{V}_{h}$}

The RT0 MFE method is given by (1.2a)-(1.2b) where the spaces $\Phi_{h}$ and $\mathbf{V}_{h}$ are specified following [46] for $d=2$ and [45] for $d=3$. The space $\Phi_{h}$ consists of piecewise constants on $\mathcal{T}_{h}$. As for the space $\mathbf{V}_{h}$, there is one basis function $\mathbf{v}_{\sigma}$ associated with each side $\sigma \in \mathcal{E}_{h}$. Let $\sigma$ be an interior 
side of the mesh $\mathcal{T}_{h}$ shared by the elements $K$ and $L$. Then the associated basis function is given by $\mathbf{v}_{\sigma}(\mathbf{x})=\frac{1}{d|K|}\left(\mathbf{x}-V_{K}\right), \mathbf{x} \in K, \mathbf{v}_{\sigma}(\mathbf{x})=\frac{1}{d|L|}\left(V_{L}-\mathbf{x}\right), \mathbf{x} \in L, \mathbf{v}_{\sigma}(\mathbf{x})=0$ otherwise, where $V_{K}$ is the vertex of $K$ opposite to $\sigma$ and $V_{L}$ the vertex of $L$ opposite to $\sigma$. The orientation of $\mathbf{v}_{\sigma}$ (the order of $K$ and $L$ ) coincides with the orientation of the normal vector $\mathbf{n}_{\sigma}$. For a boundary side $\sigma$, the support of $\mathbf{v}_{\sigma}$ only consists of the element $K \in \mathcal{T}_{h}$ such that $\sigma \in \mathcal{E}_{K}$ and $\mathbf{v}_{\sigma}(\mathbf{x})=\frac{1}{d|K|}\left(\mathbf{x}-V_{K}\right)$, $\mathbf{x} \in K$. We refer to $[16,47]$ for more details.

\subsection{Hybridization}

Let $\sigma$ be an interior side of the mesh $\mathcal{T}_{h}$ shared by the elements $K$ and $L$. The approximate fluxes $\mathbf{u}_{h}$ of (1.2a)-(1.2b) satisfy $\left.\mathbf{u}_{h}\right|_{K} \cdot \mathbf{n}_{\sigma}=\left.\mathbf{u}_{h}\right|_{L} \cdot \mathbf{n}_{\sigma}$. This constraint can be relaxed using the hybridization technique. The unconstrained flux space is given by $\mathbf{V}_{h}^{\mathrm{H}}:=\Pi_{K \in \mathcal{T}_{h}} \mathbf{V}_{h}(K)$, where $\mathbf{V}_{h}(K)$ are the local spaces on each mesh element, and the Lagrange multipliers space $\Psi_{h}$ is the space of piecewise constants on the interior sides. The hybridized version of (1.2a)-(1.2b) consists in finding $\mathbf{u}_{h} \in \mathbf{V}_{h}^{\mathrm{H}}, p_{h} \in \Phi_{h}$, and $\lambda_{h} \in \Psi_{h}$ such that

$$
\begin{aligned}
\left(\underline{\mathbf{S}}^{-1} \mathbf{u}_{h}, \mathbf{v}_{h}\right)-\left(p_{h}, \nabla \cdot \mathbf{v}_{h}\right)+\sum_{K \in \mathcal{T}_{h}}\left\langle\mathbf{v}_{h} \cdot \mathbf{n}_{K}, \lambda_{h}\right\rangle_{\partial K \backslash \partial \Omega} & =0 & & \forall \mathbf{v}_{h} \in \mathbf{V}_{h}^{\mathrm{H}}, \\
-\left(\nabla \cdot \mathbf{u}_{h}, \phi_{h}\right) & =-\left(g, \phi_{h}\right) & & \forall \phi_{h} \in \Phi_{h}, \\
\sum_{K \in \mathcal{T}_{h}}\left\langle\mathbf{u}_{h} \cdot \mathbf{n}_{K}, \psi_{h}\right\rangle_{\partial K \backslash \partial \Omega} & =0 & & \forall \psi_{h} \in \Psi_{h} .
\end{aligned}
$$

Here, $\lambda_{h}$ provides an additional approximation to the potential $p$ on the sides of the mesh. We point out that (1.2a)-(1.2b) and (3.1a)-(3.1c) lead to the same solutions $p_{h}$ and $\mathbf{u}_{h}$. Due to the different flux ansatz spaces, the algebraic vector associated with $\mathbf{u}_{h}$ in $\mathbf{V}_{h}^{\mathrm{H}}$ is different from $U$ and denoted by $U^{\mathrm{H}}$. The equations (3.1a)-(3.1c) give in matrix form

$$
\left(\begin{array}{lll}
\mathbb{A}^{\mathrm{H}} & \left(\mathbb{B}^{\mathrm{H}}\right)^{t} & \mathbb{C}^{t} \\
\mathbb{B}^{\mathrm{H}} & 0 & 0 \\
\mathbb{C} & 0 & 0
\end{array}\right)\left(\begin{array}{l}
U^{\mathrm{H}} \\
P \\
\Lambda
\end{array}\right)=\left(\begin{array}{l}
F^{\mathrm{H}} \\
G \\
0
\end{array}\right) .
$$

The importance of the hybridization (3.2) lies in the observation that the matrix $\mathbb{A}^{\mathrm{H}}$ has now an elementwise block-diagonal structure. Consequently, (3.2) can be reduced by static condensation to the problem

$$
\mathbb{Z} \Lambda=E,
$$

see [16, Section V.1]. The matrix $\mathbb{Z}$ is symmetric and positive definite with a narrow stencil.

\subsection{Relation to the Crouzeix-Raviart nonconforming finite element, finite vol- ume, multi-point flux-approximation, and mimetic methods}

Let $\Psi_{h}^{N C}$ be the Crouzeix-Raviart space, see [25]. This is the space of piecewise affine functions on $\mathcal{T}_{h}$ which are continuous in the barycenters of the interior sides and zero in the barycenters of the boundary sides. There is one basis function associated with each interior side $\sigma \in \mathcal{E}_{h}^{\text {int }}$, denoted by $\psi_{\sigma}$. It is such that $\psi_{\sigma}\left(\mathbf{x}_{\sigma}\right)=1$ and $\psi_{\sigma}\left(\mathbf{x}_{\gamma}\right)=0$ for all sides $\gamma \in \mathcal{E}_{h}$ different from $\sigma$.

The CR NCFE method for the problem (1.1a)-(1.1b) reads: find $\lambda_{h}^{\mathrm{NC}} \in \Psi_{h}^{\mathrm{NC}}$ such that

$$
\left(\underline{\mathbf{S}} \nabla \lambda_{h}^{\mathrm{NC}}, \nabla \psi_{h}\right)=\left(g, \psi_{h}\right) \quad \forall \psi_{h} \in \Psi_{h}^{\mathrm{NC}} .
$$

In a matrix setting, it can be written as

$$
\mathbb{Z} \Lambda=E .
$$


It follows from $[10,44,7,22]$ that the above matrix $\mathbb{Z}$ and the right-hand side vector $E$ coincide with the $\mathbb{Z}$ and $E$ of (3.3). Thus, the CR NCFE method (3.5) is equivalent to the hybridization (3.3) of the RT0 MFE one. Let $\sigma \in \mathcal{E}_{h}^{\text {int }}$ and denote the value of $\lambda_{h}$ of (3.1a)-(3.1c) on $\sigma$ by $\Lambda_{\sigma}$. Then we can obtain $\lambda_{h}^{\mathrm{NC}}$ of (3.4) by

$$
\lambda_{h}^{\mathrm{NC}}=\sum_{\sigma \in \mathcal{E}_{h}^{\text {int }}} \Lambda_{\sigma} \psi_{\sigma}
$$

Other relations have also been established:

Remark 3.1 (Relation to two-point FV). It has been proven in [59, 20, 57], see also the references therein, that the RTO MFE (3.1a)-(3.1c) and the two-point FV discretization coincide in two space dimensions for zero source term $\mathrm{g}$. We will in fact recover this result in Section 4.4.1 below.

Remark 3.2 (Relation to MPFA). For $d=2$ and $g=0$, it was shown in [37, 58], based on the results of [53], that the nonsymmetric MPFA O-method [1] is equivalent to the RTO MFE written with one unknown per element in the form of Section 4.1.2 below.

Remark 3.3 (Relation to MFD). In [17, Section 5.1] and [18, Example 1], it has been pointed out that the MFD method on simplicial meshes contains as one of its variants the RTO MFE method (1.2a)-(1.2b), in the sense that it leads to the same linear system (1.3).

\subsection{Local flux expressions from the Lagrange multipliers}

Following [10, 44, 22], there holds

$$
\begin{aligned}
& \left.\mathbf{u}_{h}\right|_{K}=-\left.\left.\underline{\mathbf{S}}\right|_{K} \nabla \lambda_{h}^{\mathrm{NC}}\right|_{K}+\left.\frac{\left.g_{h}\right|_{K}}{d}\left(\mathbf{x}-\mathbf{x}_{K}\right)\right|_{K}, \\
& \left.p_{h}\right|_{K}=\lambda_{h}^{\mathrm{NC}}\left(\mathbf{x}_{K}\right)+\frac{\left.g_{h}\right|_{K}}{d^{2}|K|}\left(\underline{\mathbf{S}}^{-1}\left(\mathbf{x}-\mathbf{x}_{K}\right), \mathbf{x}-\mathbf{x}_{K}\right)_{K} .
\end{aligned}
$$

Relation (3.7a) states that there exist local flux expressions from the Lagrange multipliers. Relation $(3.7 \mathrm{~b})$ means that the original potential approximation $p_{h}$ is linked to the Lagrange multipliers $\lambda_{h}$ and, additionally, to the source term $g$. Thus we can locally express both $\mathbf{u}_{h}$ and $p_{h}$ in terms of the Lagrange multipliers $\lambda_{h}$. It is, however, not obvious how to express locally $\mathbf{u}_{h}$ from $p_{h}$.

\section{Reductions to one unknown per element and local flux expres- sions}

We present here a unified framework allowing to reduce (1.3), (3.2), or (3.3) in the lowest-order MFE method equivalently to (1.7). Firstly, in Sections 4.1 and 4.2, we define local problems on patches of elements which have to be solved to give local flux expressions. Section 4.3 then shows how to express the vectors of the Lagrange multipliers $\Lambda$ or of the fluxes $U^{\mathrm{H}}$ from the new potential unknowns $\bar{P}$, and Section 4.4 gives two different ways of obtaining the final one-unknownper-element system (1.7).

\subsection{Definition of the local problems by a geometrical interpretation}

It follows from (3.6) that $\left.\lambda_{h}^{\mathrm{NC}}\right|_{K}$ for any $K \in \mathcal{T}_{h}$ is given by the $d+1$ values $\Lambda_{\sigma}$; for simplicity, following from (1.1b), we set $\Lambda_{\sigma}=0$ for all $\sigma \in \mathcal{E}_{h}^{\text {ext }}$. Let $\mathbf{z}_{K}$ be a point arbitrary in $\mathbb{R}^{d}$ but such that any $d$ of the $d+1$ side barycenters of $K$ and the point $\mathbf{z}_{K}$ do not lie in the same hyperplane. 


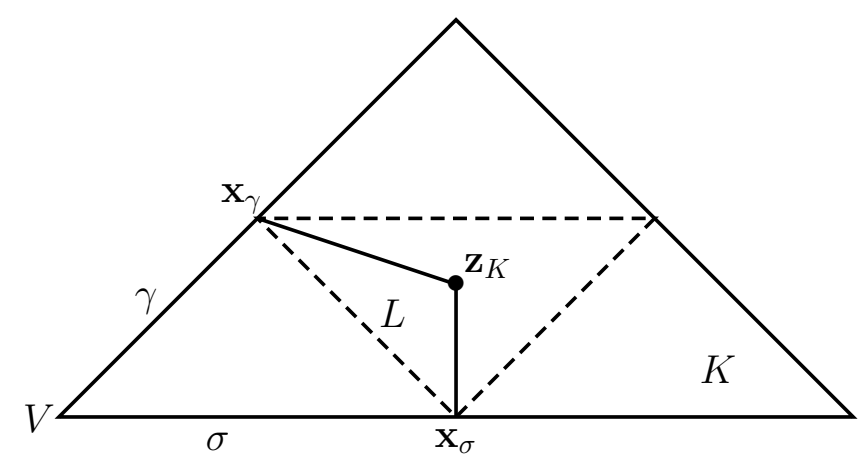

Figure 2: Triangle $K \in \mathcal{T}_{h}$ and subtriangle $L$ given by two edge midpoints $\mathbf{x}_{\sigma}$ and $\mathbf{x}_{\gamma}$ and the point $\mathbf{z}_{K}$

In two space dimensions, this means that $\mathbf{z}_{K}$ does not lie on the boundary of the dashed triangle in Figure 2. Our main idea is to use the value of $\lambda_{h}^{\mathrm{NC}}$ in the point $\mathbf{z}_{K}$ as a new unknown,

$$
\bar{P}_{K}:=\left.\lambda_{h}^{\mathrm{NC}}\right|_{K}\left(\mathbf{z}_{K}\right)
$$

Let $V$ be any of the vertices of $K$ and let a new simplex $L$ be given by the side barycenters $\mathbf{x}_{\sigma}, \sigma \in \mathcal{E}_{V, K}$, and by the point $\mathbf{z}_{K}$, see Figure 2 (here the points are denoted by $\mathbf{x}_{\sigma}, \mathbf{x}_{\gamma}$, and $\mathbf{z}_{K}$ ). Denote by $\varphi_{\sigma}, \sigma \in \mathcal{E}_{V, K}$, and by $\varphi_{K}$ the Lagrange basis functions associated with the vertices of $L$; we consider $\varphi_{\sigma}$ and $\varphi_{K}$ supported on $K$. Then, using (3.7a), we get

$$
\left.\mathbf{u}_{h}\right|_{K}=-\left.\underline{\mathbf{S}}\right|_{K} \nabla\left(\sum_{\sigma \in \mathcal{E}_{V, K}} \Lambda_{\sigma} \varphi_{\sigma}+\bar{P}_{K} \varphi_{K}\right)+\left.\frac{\left.g_{h}\right|_{K}}{d}\left(\mathbf{x}-\mathbf{x}_{K}\right)\right|_{K} .
$$

Let now a vertex $V \in \mathcal{V}_{h}$ be fixed and consider the patch $\mathcal{T}_{V}$ of elements of $\mathcal{T}_{h}$ which share $V$. We refer to Figure 1 for examples. Consider (3.1c) for $\psi_{h}$ associated with the internal sides $\mathcal{E}_{V}^{\text {int }}$ of the patch $\mathcal{T}_{V}$. This gives rise to the following local problem: given $\bar{P}_{K}, K \in \mathcal{T}_{V}$, find $\Lambda_{\gamma}, \gamma \in \mathcal{E}_{V}^{\text {int }}$, such that (4.2) holds together with

$$
\sum_{K \in \mathcal{T}_{\sigma}}\left\langle\mathbf{u}_{h} \cdot \mathbf{n}_{K}, 1\right\rangle_{\sigma}=0 \quad \forall \sigma \in \mathcal{E}_{V}^{\mathrm{int}} .
$$

Suppose that this square linear system is well-posed. Then we can express the Lagrange multipliers $\Lambda_{\gamma}$ inside the patch $\mathcal{T}_{V}$ as a function of the new potentials $\bar{P}_{K}$ in the patch $\mathcal{T}_{V}$ and of the sources $g_{h}$ in the patch $\mathcal{T}_{V}$. Considering (4.2), we can also get the fluxes $\mathbf{u}_{h}$ in the whole patch $\mathcal{T}_{V}$ as a function of $\bar{P}_{K}$, i.e., we obtain local flux expressions from the new potentials $\bar{P}_{K}$.

Remark 4.1 (Construction principle and MPFA). Considering a patch of (sub)elements, supposing a piecewise affine, possibly nonconforming, potential approximation, imposing the normal flux continuity, and solving a local linear system on the patch is also the principle of the MPFA method [1, 3, 19], cf. also [28, 41, 14, 56, 42]. In the MPFA method, the fluxes on patches of (sub)elements are locally recovered from the potentials strongly by $\mathbf{u}_{h}:=-\underline{\mathbf{S}} \nabla p_{h}$. The difference of our approach is that we recover the fluxes from the potentials by a weak, variationally consistent formulation, following both the potential-flux relation $\mathbf{u}=-\underline{\mathbf{S}} \nabla p$ and the divergence constraint $\nabla \cdot \mathbf{u}=g$, see also [53, Remarks 2.2 and 2.3].

Let us now elaborate on (4.2)-(4.3). First recall that (4.3) can be equivalently rewritten as

$$
\sum_{K \in \mathcal{T}_{\sigma}}\left\langle\mathbf{u}_{h} \cdot \mathbf{n}_{K}, \psi_{\sigma}\right\rangle_{\partial K}=0 \quad \forall \sigma \in \mathcal{E}_{V}^{\text {int }}
$$



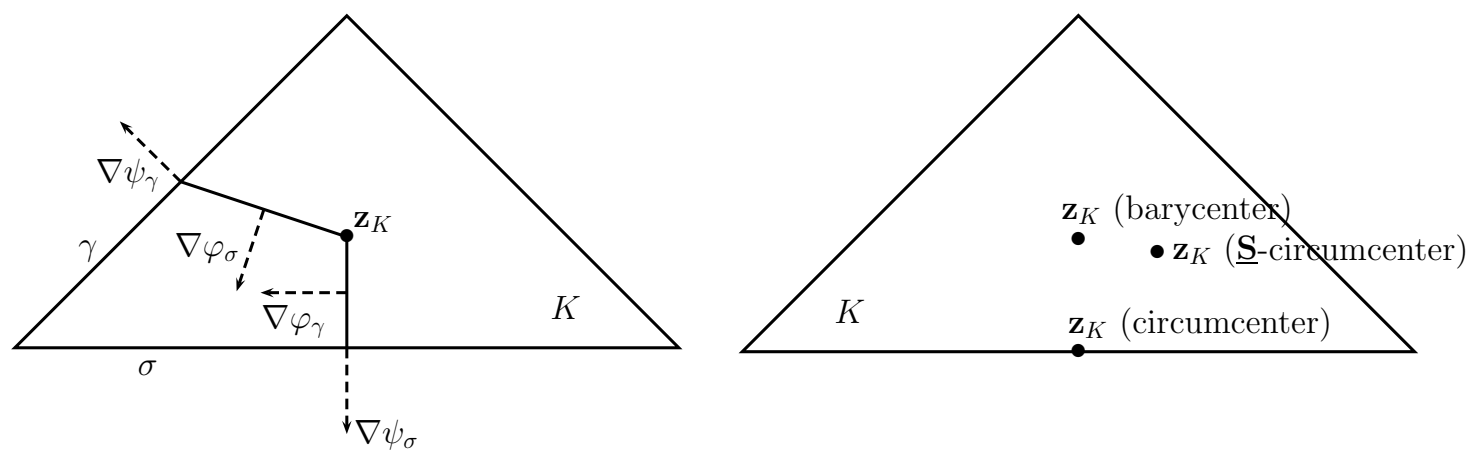

Figure 3: Gradients of the basis functions (left) and different evaluation points $\mathbf{z}_{K}$ (right) in an element $K \in \mathcal{T}_{h}$

Here $\psi_{\sigma}$ are the CR nonconforming basis functions, see Section 3.3. This follows by the facts that $\mathbf{u}_{h} \cdot \mathbf{n}_{K}$ is constant on any $\sigma \in \mathcal{E}_{K},\left\langle 1, \psi_{\sigma}\right\rangle_{\sigma}=|\sigma|$, and $\left\langle 1, \psi_{\sigma}\right\rangle_{\gamma}=0$ for any side $\gamma$ different from $\sigma$, so that $\left\langle\mathbf{u}_{h} \cdot \mathbf{n}_{K}, 1\right\rangle_{\sigma}=\left\langle\mathbf{u}_{h} \cdot \mathbf{n}_{K}, \psi_{\sigma}\right\rangle_{\sigma}=\left\langle\mathbf{u}_{h} \cdot \mathbf{n}_{K}, \psi_{\sigma}\right\rangle_{\partial K}$. From this equality, using the Green theorem, we have

$$
\sum_{K \in \mathcal{T}_{\sigma}}\left\{\left(\nabla \cdot \mathbf{u}_{h}, \psi_{\sigma}\right)_{K}+\left(\mathbf{u}_{h}, \nabla \psi_{\sigma}\right)_{K}\right\}=0 \quad \forall \sigma \in \mathcal{E}_{V}^{\mathrm{int}}
$$

It follows from (1.2b) and the definition of $\mathbf{V}_{h}(K)$ in Section 3 that $\left.\left(\nabla \cdot \mathbf{u}_{h}\right)\right|_{K}=\left.g_{h}\right|_{K}$. For the other term in the above relation, we employ (4.2). Notice that

$$
\left(\mathbf{x}-\mathbf{x}_{K}, \nabla \psi_{\sigma}\right)_{K}=0,
$$

as $\left.\left(\nabla \psi_{\sigma}\right)\right|_{K}$ is a constant vector and $\mathbf{x}_{K}$ is the barycenter of $K$. Extend the notation $\varphi_{\sigma}$ from (4.2) to a Lagrange basis function supported on all $K \in \mathcal{T}_{V}$ such that $\sigma \in \mathcal{E}_{K}$. The gradients of these basis function are illustrated in the left part of Figure 3. We then see that (4.2)-(4.3) is equivalent to the following problem: given $\bar{P}_{K}, K \in \mathcal{T}_{V}$, find $\Lambda_{\gamma}, \gamma \in \mathcal{E}_{V}^{\text {int }}$, such that

$$
\sum_{K \in \mathcal{T}_{\sigma}} \sum_{\gamma \in \mathcal{E}_{V}^{\mathrm{int}}} \Lambda_{\gamma}\left(\underline{\mathbf{S}} \nabla \varphi_{\gamma}, \nabla \psi_{\sigma}\right)_{K}=\sum_{K \in \mathcal{T}_{\sigma}}\left\{\left(g, \psi_{\sigma}\right)_{K}-\bar{P}_{K}\left(\underline{\mathbf{S}} \nabla \varphi_{K}, \nabla \psi_{\sigma}\right)_{K}\right\} \quad \forall \sigma \in \mathcal{E}_{V}^{\mathrm{int}} .
$$

We point out that (4.5) is a Petrov-Galerkin problem, as the basis functions $\psi_{\sigma}$ of the test space are different from the basis functions $\varphi_{\gamma}$ of the trial space.

The matrix form of (4.5) writes: given $\bar{P}_{V}:=\left\{\bar{P}_{K}\right\}_{K \in \mathcal{T}_{V}}$, find $\Lambda_{V}^{\text {int }}:=\left\{\Lambda_{\gamma}\right\}_{\gamma \in \mathcal{E}_{V}^{\text {int }}}$ such that

$$
\mathbb{M}_{V} \Lambda_{V}^{\mathrm{int}}=\widetilde{G}_{V}-\mathbb{J}_{V} \bar{P}_{V}
$$

where

$$
\begin{aligned}
\left(\mathbb{M}_{V}\right)_{\sigma, \gamma} & :=\sum_{K \in \mathcal{T}_{\sigma}}\left(\underline{\mathbf{S}} \nabla \varphi_{\gamma}, \nabla \psi_{\sigma}\right)_{K}, \\
\left(\widetilde{G}_{V}\right)_{\sigma} & :=\sum_{K \in \mathcal{T}_{\sigma}}\left(g, \psi_{\sigma}\right)_{K}, \\
\left(\mathbb{J}_{V}\right)_{\sigma, K} & :=-\left(\underline{\mathbf{S}} \nabla \varphi_{K}, \nabla \psi_{\sigma}\right)_{K} .
\end{aligned}
$$

So far, we have not specified the choice of the points $\mathbf{z}_{K}$. Three different choices of $\mathbf{z}_{K}$ have been already studied in the literature; however in none of the approaches an abstract framework was provided. 


\subsection{1 $\quad$ S-circumcenter as the evaluation point}

Problem (4.5) was first studied in [59, 20] for two space dimensions and in [57] for particular meshes in three space dimensions. One supposes here the existence of a point $\mathbf{z}_{K}$ such that $\left.\underline{\mathbf{S}}\right|_{K} \nabla \varphi_{\gamma} \cdot \nabla \psi_{\sigma}=0$ and $\left.\underline{\mathbf{S}}\right|_{K} \nabla \varphi_{\sigma} \cdot \nabla \psi_{\gamma}=0$ for all $K \in \mathcal{T}_{h}$, with the notation of the left part of Figure 3, which leads to diagonal matrices $\mathbb{M}_{V}$. Such a point always exists in two space dimensions and is called the $\underline{\mathbf{S}}$-circumcenter of $K$; the whole mesh $\mathcal{T}_{h}$ then becomes "S-orthogonal grid" in the terminology of [1]. When $\left.\underline{\mathbf{S}}\right|_{K}=\mathbb{I} s_{K}, \mathbf{z}_{K}$ gets the circumcenter of $K$. The right part of Figure 3 gives an illustration for a diffusion tensor given by $\underline{\mathbf{S}}=\left(\begin{array}{ll}0.7236 & 0.3804 \\ 0.3804 & 0.4764\end{array}\right)$.

In the approach of this section, no local linear system needs to be solved and one always obtains a two-point flux expression. The choice of the evaluation point $\mathbf{z}_{K}$ depends on the diffusion tensor $\underline{\mathbf{S}}$ but not on the local mesh form. Let $d=2$ and consider a patch $\mathcal{T}_{V}$ as in Section 4.1 and a side $\sigma \in \mathcal{E}_{V}^{\text {int }}$. Then, from (4.5), we get

$$
\Lambda_{\sigma}=\frac{\left(g, \psi_{\sigma}\right)_{K}+\left(g, \psi_{\sigma}\right)_{L}-\bar{P}_{K}\left(\underline{\mathbf{S}} \nabla \varphi_{K}, \nabla \psi_{\sigma}\right)_{K}-\bar{P}_{L}\left(\underline{\mathbf{S}} \nabla \varphi_{L}, \nabla \psi_{\sigma}\right)_{L}}{\left(\underline{\mathbf{S}} \nabla \varphi_{\sigma}, \nabla \psi_{\sigma}\right)_{K}+\left(\underline{\mathbf{S}} \nabla \varphi_{\sigma}, \nabla \psi_{\sigma}\right)_{L}} .
$$

By the same reasoning as in Section 4.1, we come to

$$
\left\langle\mathbf{u}_{h} \cdot \mathbf{n}_{K}, 1\right\rangle_{\sigma}=-\Lambda_{\sigma}\left(\underline{\mathbf{S}} \nabla \varphi_{\sigma}, \nabla \psi_{\sigma}\right)_{K}+\left(g, \psi_{\sigma}\right)_{K}-\bar{P}_{K}\left(\underline{\mathbf{S}} \nabla \varphi_{K}, \nabla \psi_{\sigma}\right)_{K} .
$$

We now use the fact that

$$
\left.\underline{\mathbf{S}}\right|_{K} \nabla \varphi_{K} \cdot \nabla \psi_{\sigma}=-\left.\underline{\mathbf{S}}\right|_{K} \nabla \varphi_{\sigma} \cdot \nabla \psi_{\sigma}
$$

which follows from the basis functions orthogonalities and from the fact that $\varphi_{\sigma}, \varphi_{\gamma}$, and $\varphi_{K}$ form a partition of unity on $K$. Denote

$$
\alpha_{K, \sigma}:=\left(\underline{\mathbf{S}} \nabla \varphi_{\sigma}, \nabla \psi_{\sigma}\right)_{K}
$$

It can be checked that this expression does not depend, for a fixed $K \in \mathcal{T}_{h}$ and $\sigma \in \mathcal{E}_{K}$, on the vertex $V$ of $\sigma ;(4.11)$ coincides with [57, equation (31)]. If $\left.\underline{\mathbf{S}}\right|_{K}=\mathbb{I} s_{K}$, it follows that

$$
\alpha_{K, \sigma}=\operatorname{sgn}\left(\mathbf{z}_{K}\right) \frac{s_{K}|\sigma|}{\left|\mathbf{z}_{K}-\mathbf{x}_{\sigma}\right|},
$$

where $\operatorname{sgn}\left(\mathbf{z}_{K}\right)=1$ if $\mathbf{z}_{K} \in K$ and -1 otherwise. Using the analogous notation $\alpha_{L, \sigma}$ for the element $L,(4.8)$ can be rewritten as

$$
\Lambda_{\sigma}=\frac{\left(g, \psi_{\sigma}\right)_{K}+\left(g, \psi_{\sigma}\right)_{L}+\bar{P}_{K} \alpha_{K, \sigma}+\bar{P}_{L} \alpha_{L, \sigma}}{\alpha_{K, \sigma}+\alpha_{L, \sigma}} .
$$

For $g=0$, this is the standard two-point FV formula, cf. the first equation on page 13 of [31]. Note however that, as discussed in [59, Section 4], this is no more the case when $g \neq 0$, and the coefficients $\alpha_{K, \sigma}$ can be negative. Inserting the expression for $\Lambda_{\sigma}$ from (4.12) to (4.9), we obtain

$$
\left\langle\mathbf{u}_{h} \cdot \mathbf{n}_{K}, 1\right\rangle_{\sigma}=\frac{\alpha_{K, \sigma} \alpha_{L, \sigma}}{\alpha_{K, \sigma}+\alpha_{L, \sigma}}\left(\bar{P}_{K}-\bar{P}_{L}\right)+\left(g, \psi_{\sigma}\right)_{K}-\frac{\alpha_{K, \sigma}}{\alpha_{K, \sigma}+\alpha_{L, \sigma}}\left(\left(g, \psi_{\sigma}\right)_{K}+\left(g, \psi_{\sigma}\right)_{L}\right),
$$

i.e., the formula (34) from [57]. When $g=0$ and $\underline{\mathbf{S}}$ is scalar, (4.13) is nothing but the standard two-point FV flux with harmonic averaging of the diffusion coefficient, cf. the second equation on page 13 of [31]. The influence of the source term $g$ only disappears in very particular situations; a 

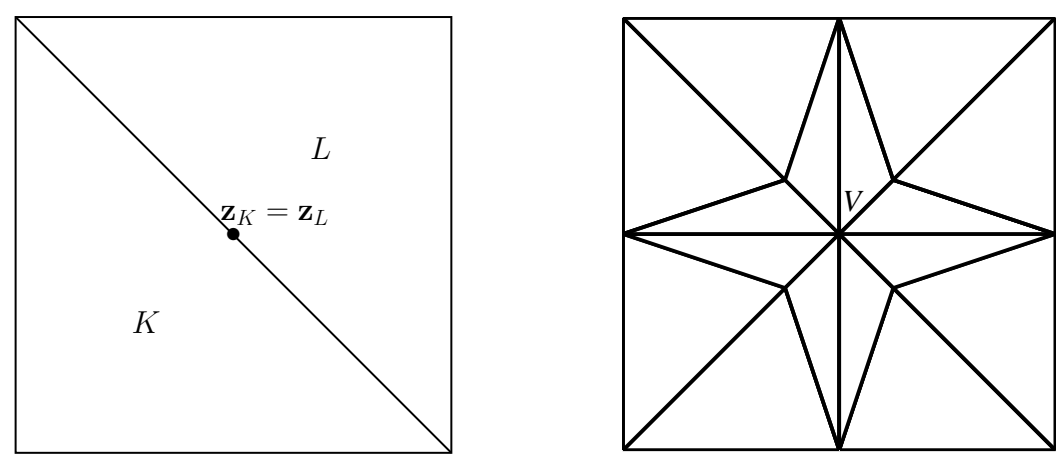

Figure 4: Two triangles cutting a square for circumcenter evaluation points (left) and an example of a patch $\mathcal{T}_{V}$ where the matrix $\mathbb{M}_{V}$ is singular for barycenter evaluation points (right)

sufficient condition is, for example, $\underline{\mathbf{S}}=\mathbb{I}$ and $\left.g_{h}\right|_{K}|K|\left|\mathbf{z}_{K}-\mathbf{x}_{\sigma}\right| \operatorname{sgn}\left(\mathbf{z}_{K}\right)=\left.g_{h}\right|_{L}|L|\left|\mathbf{z}_{L}-\mathbf{x}_{\sigma}\right| \operatorname{sgn}\left(\mathbf{z}_{L}\right)$. For $\sigma \in \mathcal{E}_{h}^{\text {ext }}$, one similarly obtains

$$
\left\langle\mathbf{u}_{h} \cdot \mathbf{n}_{K}, 1\right\rangle_{\sigma}=\alpha_{K, \sigma} \bar{P}_{K}+\left(g, \psi_{\sigma}\right)_{K} .
$$

The present approach can degenerate. This happens when $\mathbf{z}_{K}$ coincides with one of the side barycenters $\mathbf{x}_{\sigma}$, as illustrated in Figure 4, left part. Then, with the notation of Figure 2, the measure of the subtriangle $L$ becomes $0,|L|=0$. Consequently, one obtains $\left|\nabla \varphi_{\sigma}\right|=\infty$. This situation requires a specific treatment, see Section 4.4.1 below.

\subsubsection{Barycenter as the evaluation point}

The choice of the barycenter $\mathbf{z}_{K}=\mathbf{x}_{K}$, cf. the right part of Figure 3 , is related to the approach studied in [53]. It turns out that this choice allows for a wider variety of meshes for which the local problems (4.6) are well-posed and that it works in all space dimensions. The matrix $\mathbb{M}_{V}$ is, in general, not diagonal. This leads to the necessity to solve local linear systems and to a multi-point flux expression where the fluxes depend on the potentials $\bar{P}_{K}$ of the whole patch $\mathcal{T}_{V}$. The choice of the evaluation point $\mathbf{z}_{K}$ depends neither on the diffusion tensor $\underline{\mathbf{S}}$, nor on the local mesh form. This approach can, however, lead to a singular local condensation matrix $\mathbb{M}_{V}$. This happens, e.g., for $\underline{\mathbf{S}}=\mathbb{I}$ and the patch $\mathcal{T}_{V}$ illustrated in Figure 4, right part.

In [53], one expresses $\left.\mathbf{u}_{h}\right|_{\mathcal{T}_{V}}$ directly from the original unknowns $P_{K}:=\left.p_{h}\right|_{K}, K \in \mathcal{T}_{V}$. When $g=0$, we have from (3.7b) $P_{K}=\bar{P}_{K}=\lambda_{h}^{\mathrm{NC}}\left(\mathbf{z}_{K}\right)$ and thus the local problems (4.5) coincide with those given in [53]. When $g \neq 0$, it follows from (3.7b) that $P_{K}$ and $\bar{P}_{K}=\lambda_{h}^{\mathrm{NC}}\left(\mathbf{z}_{K}\right)$ only differ by $\frac{\left.g_{h}\right|_{K}}{d^{2}|K|}\left(\underline{\mathbf{S}}^{-1}\left(\mathbf{x}-\mathbf{x}_{K}\right), \mathbf{x}-\mathbf{x}_{K}\right)_{K}$. Consequently, the local problems (4.5) and those of [53] can still be written in the form (4.6) with the same local matrices $\mathbb{M}_{V}$ given by (4.7a) and only differ by the vectors $\bar{P}_{V}$ and the right-hand side.

Remark 4.2 (Singular matrices in the MPFA method). Recall from [37, 58] that the RTO MFE with one unknown per element and the nonsymmetric MPFA O-method are equivalent for $d=2$ and $g=0$, cf. Remark 3.2. Thus, this variant of the MPFA also gives rise to a singular matrix $\mathbb{M}_{V}$ for the mesh of Figure 4, right part.

\subsubsection{Mesh- and diffusion tensor-dependent evaluation point}

In the framework of the CR NCFE method, a new idea has been proposed in [55] for the solution of the local problems (4.5). It consists in choosing the evaluation point according to the mesh $\mathcal{T}_{h}$ 
and the diffusion tensor $\underline{\mathbf{S}}$. The choice of the points $\mathbf{z}_{K}$ is done locally in order to: a) ensure the well-posedness of the local problems (4.5); b) influence the properties of the local matrices $\mathbb{M}_{V}$; c) influence the properties of the final global system matrices (see Sections 4.4.1 and 4.4.2 below). We refer to $[31,2,27]$ for some other examples of locally influencing the final system properties.

\subsection{Definition of the local problems by an algebraic interpretation}

We now generalize the approach of Section 4.1 in a purely algebraic way, following [55].

\subsubsection{Potentials viewpoint}

Let $K \in \mathcal{T}_{h}$, let $\Lambda_{K}=\left\{\Lambda_{\gamma}\right\}_{\gamma \in \mathcal{E}_{K}^{\text {int }}}$ be the vector of the Lagrange multipliers associated with the interior sides of the element $K$, and let $\mathbb{N}_{K}$ be a $1 \times\left|\mathcal{E}_{K}^{\text {int }}\right|$ matrix. We define the vector $\bar{P}_{K}$ in a purely algebraic way by

$$
\mathbb{N}_{K} \Lambda_{K}=\bar{P}_{K}
$$

Whenever additionally the constraint

$$
\sum_{\sigma \in \mathcal{E}_{K}}\left(\mathbb{N}_{K}\right)_{\sigma}=1
$$

holds for all interior mesh elements, (4.15) allows for a geometrical interpretation in the sense of Section 4.1: then the entries $\left(\mathbb{N}_{K}\right)_{\sigma}$ take the values of the CR basis functions $\psi_{\sigma}$ at the evaluation points $\mathbf{z}_{K}$. As an example, in Section 4.1.2, all entries of $\mathbb{N}_{K}$ are equal to $1 /(d+1)$, whereas the matrix $\mathbb{N}_{K}$ for Section 4.1.1 is specified in [57, equation (30)].

Now consider a vertex $V \in \mathcal{V}_{h}$ and (4.15) on all elements $K \in \mathcal{T}_{V}^{\text {ext }}$ (recall that Figure 1 gives an illustration of the different sets). This gives $\left|\mathcal{T}_{V}^{\text {ext }}\right|=\left|\mathcal{E}_{V}^{\text {ext }}\right|$ equations involving the unknowns $\Lambda_{V}:=\left\{\Lambda_{\gamma}\right\}_{\gamma \in \mathcal{E}_{V}}$, with the matrix $\mathbb{N}_{V}$ employing the element matrices $\mathbb{N}_{K}$ :

$$
\mathbb{N}_{V} \Lambda_{V}=\bar{P}_{V}^{\text {ext }}
$$

Consider next the lines in (3.3) associated with $\gamma \in \mathcal{E}_{V}^{\text {int }}$. Denoting $\mathbb{Z}_{V}$ the corresponding submatrix of $\mathbb{Z}$ and setting $E_{V}^{\text {int }}:=E_{\gamma \in \mathcal{E}_{V}^{\text {int }}}$, this gives a second rectangular linear system, with the number of rows given by $\left|\mathcal{E}_{V}^{\text {int }}\right|$ and the number of columns is given by $\left|\mathcal{E}_{V}\right|$,

$$
\mathbb{Z}_{V} \Lambda_{V}=E_{V}^{\mathrm{int}}
$$

Combining (4.17) and (4.19), we obtain a square linear system

$$
\left(\begin{array}{c}
\mathbb{Z}_{V} \\
\mathbb{N}_{V}
\end{array}\right) \Lambda_{V}=\left(\begin{array}{c}
E_{V}^{\text {int }} \\
\bar{P}_{V}^{\text {ext }}
\end{array}\right)
$$

As the equations (4.18) are taken from the well-posed system (3.3), they are linearly independent. Thus the well-posedness of (4.19) only depends on the matrix $\mathbb{N}_{V}$. Clearly, in a still more general fashion than in Section 4.1.3, the well-posedness of (4.19) can be controlled in function of the local constellation (mesh $\mathcal{T}_{h}$, tensor $\underline{\mathbf{S}}$ ). 


\subsubsection{Fluxes viewpoint}

Starting from the hybridized formulation (3.2) instead of (3.3) and following the same general idea as in Section 4.2.1, we can also proceed as follows: consider a vertex $V \in \mathcal{V}_{h}$ and the lines in (3.2) associated with all the elements of the patch $\mathcal{T}_{V}$ and their sides for the first block row, all the elements of the patch $\mathcal{T}_{V}$ for the second block row, and all the interior sides of the patch $\mathcal{T}_{V}$ for the last block row. Set $U_{V}^{\mathrm{H}}$ for all the elements of the patch $\mathcal{T}_{V}$ and their sides, $P_{V}$ for all the elements of the patch $\mathcal{T}_{V}$, and $\Lambda_{V}$ for the sides from $\mathcal{E}_{V}$. This gives the following local rectangular linear system:

$$
\left(\begin{array}{lll}
\mathbb{A}_{V}^{\mathrm{H}} & \left(\mathbb{B}_{V}^{\mathrm{H}}\right)^{t} & \mathbb{C}_{V}^{t} \\
\mathbb{B}_{V}^{\mathrm{H}} & 0 & 0 \\
\mathbb{C}_{V} & 0 & 0
\end{array}\right)\left(\begin{array}{c}
U_{V}^{\mathrm{H}} \\
P_{V} \\
\Lambda_{V}
\end{array}\right)=\left(\begin{array}{c}
F_{V}^{\mathrm{H}} \\
G_{V} \\
0_{V}
\end{array}\right) .
$$

Combing (4.20) with (4.17) gives a local square linear system with $(d+1)\left|\mathcal{T}_{V}\right|+\left|\mathcal{T}_{V}\right|+\left|\mathcal{E}_{V}^{\text {int }}\right|+\left|\mathcal{T}_{V}^{\text {ext }}\right|$ equations and unknowns

$$
\left(\begin{array}{lll}
\mathbb{A}_{V}^{\mathrm{H}} & \left(\mathbb{B}_{V}^{\mathrm{H}}\right)^{t} & \mathbb{C}_{V}^{t} \\
\mathbb{B}_{V}^{\mathrm{H}} & 0 & 0 \\
\mathbb{C}_{V} & 0 & 0 \\
0 & 0 & \mathbb{N}_{V}
\end{array}\right)\left(\begin{array}{c}
U_{V}^{\mathrm{H}} \\
P_{V} \\
\Lambda_{V}
\end{array}\right)=\left(\begin{array}{c}
F_{V}^{\mathrm{H}} \\
G_{V} \\
0_{V} \\
\bar{P}_{V}^{\text {ext }}
\end{array}\right)
$$

Thus we can also obtain the fluxes in the whole patch $\mathcal{T}_{V}$ as a function of the potentials.

\subsection{Recovery of $\Lambda$ and $U^{\mathrm{H}}$ in terms of $\bar{P}$}

Let $V \in \mathcal{V}_{h}$ be a vertex and $\mathcal{T}_{V}$ the corresponding patch. By any of the approaches of Sections 4.14.2 , if the corresponding local problems (4.6), (4.19), or (4.21) are well-posed, we obtain local expressions of the Lagrange multipliers $\Lambda_{V}^{\text {int }}\left(\Lambda_{V}\right)$ and/or local expressions of the fluxes $U_{V}^{\mathrm{H}}$ from the potentials $\bar{P}_{V}$ (and sources in $\mathcal{T}_{V}$ ). In particular, we infer from (4.6)

$$
\Lambda_{V}^{\mathrm{int}}=\left(\mathbb{M}_{V}\right)^{-1}\left(\widetilde{G}_{V}-\mathbb{J}_{V} \bar{P}_{V}\right)
$$

and from (4.19)

$$
\Lambda_{V}=\left(\begin{array}{c}
\mathbb{Z}_{V} \\
\mathbb{N}_{V}
\end{array}\right)^{-1}\left(\begin{array}{c}
E_{V}^{\text {int }} \\
\bar{P}_{V}^{\text {ext }}
\end{array}\right)
$$

Recall that the system matrices in (4.22) and (4.23) are square. A similar expression can be obtained for $U_{V}^{\mathrm{H}}$ from all (4.6), (4.19), or (4.21).

We now give details on the construction (4.6). Consider (4.22) and run through all vertices of the mesh $\mathcal{T}_{h}$. For every vertex $V$, we have one expression for $\Lambda_{V}^{\text {int }}$. All these different expressions have to lead to the same values of the Lagrange multipliers $\Lambda$, since the vector $\Lambda$ is the unique solution of (3.3). Thus, we are free to associate a weight $w_{V, \sigma}$ to the expression of $\Lambda_{\sigma}$ from every patch $\mathcal{T}_{V}$ where the side $\sigma$ is such that $\Lambda_{\sigma}$ is the unknown in the local problem, and combine these expressions with these weights. The only condition is that, for every side $\sigma$, the sum of all its weights is equal to one. Let $V \in \mathcal{V}_{h}$. We define a mapping $\Upsilon_{V}: \mathbb{R}^{\left|\mathcal{E}_{V}^{\text {int }}\right|} \rightarrow \mathbb{R}^{\left|\mathcal{E}_{h}^{\text {int }}\right|}$, extending a vector $\Lambda_{V}^{\text {int }}=\left\{\Lambda_{\sigma}\right\}_{\sigma \in \mathcal{E}_{V}^{\text {int }}}$ of values associated with the sides from $\mathcal{E}_{V}^{\text {int }}$ to a vector of values associated with all the interior sides $\mathcal{E}_{h}^{\text {int }}$ by

$$
\left[\Upsilon_{V}\left(\Lambda_{V}^{\text {int }}\right)\right]_{\sigma}:=\left\{\begin{array}{cl}
\Lambda_{\sigma} & \text { if } \sigma \in \mathcal{E}_{V}^{\text {int }} \\
0 & \text { if } \sigma \notin \mathcal{E}_{V}^{\text {int }}
\end{array}\right.
$$


Let next $\mathbb{W}_{V}$ be a diagonal matrix of size $\left|\mathcal{E}_{V}^{\text {int }}\right| \times\left|\mathcal{E}_{V}^{\text {int }}\right|$, with the entries given by the weights $w_{V, \sigma}$. With these notations, we have

$$
\sum_{V \in \mathcal{V}_{h}} \Upsilon_{V}\left(\mathbb{W}_{V} \Lambda_{V}^{\mathrm{int}}\right)=\Lambda
$$

We now introduce a mapping $\Upsilon_{V}: \mathbb{R}^{\left|\mathcal{E}_{V}^{\text {int }}\right| \times\left|\mathcal{E}_{V}^{\text {int }}\right|} \rightarrow \mathbb{R}^{\left|\mathcal{E}_{h}^{\text {int }}\right| \times\left|\mathcal{E}_{h}^{\text {int }}\right|}$ (with the same name as the previous one, since there is no possibility of confusion), extending a local matrix $\mathbb{M}_{V}$ to a full-size one by zeros by

$$
\left[\Upsilon_{V}\left(\mathbb{M}_{V}\right)\right]_{\sigma, \gamma}:=\left\{\begin{array}{cl}
\left(\mathbb{M}_{V}\right)_{\sigma, \gamma} & \text { if } \sigma \in \mathcal{E}_{V}^{\text {int }} \text { and } \gamma \in \mathcal{E}_{V}^{\text {int }} \\
0 & \text { if } \sigma \notin \mathcal{E}_{V}^{\text {int }} \text { or } \gamma \notin \mathcal{E}_{V}^{\text {int }}
\end{array}\right.
$$

We finally define a mapping $\Theta_{V}: \mathbb{R}^{\left|\mathcal{E}_{V}^{\text {int }}\right| \times\left|\mathcal{T}_{V}\right|} \rightarrow \mathbb{R}^{\left|\mathcal{E}_{h}^{\text {int }}\right| \times\left|\mathcal{T}_{h}\right|}$, filling a full-size representation of a matrix $\mathbb{J}_{V}$ by zeros on the rows associated with the sides that are not from $\mathcal{E}_{V}^{\text {int }}$ and on the columns associated with the elements that are not from $\mathcal{T}_{V}$,

$$
\left[\Theta_{V}\left(\mathbb{J}_{V}\right)\right]_{\sigma, K}:=\left\{\begin{array}{cl}
\left(\mathbb{J}_{V}\right)_{\sigma, K} & \text { if } \sigma \in \mathcal{E}_{V}^{\text {int }} \text { and } K \in \mathcal{T}_{V} \\
0 & \text { if } \sigma \notin \mathcal{E}_{V}^{\text {int }} \text { or } K \notin \mathcal{T}_{V}
\end{array} .\right.
$$

With these notations, we obtain from (4.22)

$$
\begin{aligned}
\Upsilon_{V}\left(\mathbb{W}_{V} \Lambda_{V}^{\text {int }}\right) & =\Upsilon_{V}\left(\mathbb{W}_{V}\left(\mathbb{M}_{V}\right)^{-1}\left(\widetilde{G}_{V}-\mathbb{J}_{V} \bar{P}_{V}\right)\right) \\
& =\Upsilon_{V}\left(\mathbb{W}_{V}\left(\mathbb{M}_{V}\right)^{-1}\right) \widetilde{G}-\Theta_{V}\left(\mathbb{W}_{V}\left(\mathbb{M}_{V}\right)^{-1} \mathbb{J}_{V}\right) \bar{P}
\end{aligned}
$$

Now, employing (4.24), we finally come to

$$
\Lambda=\widetilde{\mathbb{M}}^{\text {inv }} \widetilde{G}-\mathbb{M}^{\text {inv }} \bar{P}
$$

with

$$
\widetilde{\mathbb{M}}^{\text {inv }}:=\sum_{V \in \mathcal{V}_{h}} \Upsilon_{V}\left(\mathbb{W}_{V}\left(\mathbb{M}_{V}\right)^{-1}\right), \quad \mathbb{M}^{\mathrm{inv}}:=\sum_{V \in \mathcal{V}_{h}} \Theta_{V}\left(\mathbb{W}_{V}\left(\mathbb{M}_{V}\right)^{-1} \mathbb{J}_{V}\right)
$$

A similar procedure as above can be applied for the local problems (4.19) or (4.21).

In the same way, in (4.6), (4.19), or (4.21), the fluxes can be expressed. We then arrive at the equivalent of (4.25) in the form

$$
U^{\mathrm{H}}=\widetilde{\mathbb{O}}^{\text {inv }} G-\mathbb{O}^{\text {inv }} \bar{P} .
$$

Let us stress that $\mathbb{M}^{\text {inv }}, \widetilde{\mathbb{M}}^{\text {inv }}, \mathbb{O}^{\text {inv }}$, and $\widetilde{\mathbb{O}}^{\text {inv }}$ are fully computable and sparse matrices, obtained by a weighted combination of the inverses of the local matrices. We would finally like to mention here that in the numerical experiments of Section 5, we only use the approach of Section 4.1, with all the weights $w_{V, \sigma}$ equal to $1 / d$.

\subsection{Prescribing the final system for the potentials $\bar{P}$ only}

We now want to use the above developments in order to write a global system of the form (1.7). It turns out that two different approaches can be used.

\subsubsection{Using the equilibrium of the fluxes}

The first possibility is to insert (4.26) into the second block equation of (3.2) which yields

$$
-\mathbb{B}^{\mathrm{H}} \mathbb{O}^{\text {inv }} \bar{P}=G-\mathbb{B}^{\mathrm{H}} \widetilde{\mathbb{O}}^{\text {inv }} G,
$$


i.e., (1.7) with the square matrix $\mathbb{S}:=-\mathbb{B}^{\mathrm{H}} \mathbb{O}^{\text {inv }}$ and the right-hand side vector $H:=G-\mathbb{B}^{\mathrm{H}} \widetilde{\mathbb{O}}^{\text {inv }} G$. Remark that here, the second block equation of (3.2) is used repeatedly.

The approach of Section 4.1.1 allows to state the matrix $\mathbb{S}$ explicitly. Fix $K \in \mathcal{T}_{h}$ for $d=2$ and recall that (4.11), for $\sigma \in \mathcal{E}_{K} \cap \mathcal{E}_{h}^{\text {int }}$, takes the same values when expressed from the two patches such that $\sigma \in \mathcal{E}_{V}^{\text {int }}$. Thus, the weights $w_{V, \sigma}$ of Section 4.3 have no influence in this case and can be chosen arbitrarily. Plugging (4.13)-(4.14) into $\left(\nabla \cdot \mathbf{u}_{h}, 1\right)_{K}=\sum_{\sigma \in \mathcal{E}_{K}}\left\langle\mathbf{u}_{h} \cdot \mathbf{n}_{K}, 1\right\rangle_{\sigma}=(g, 1)_{K}$ then gives (1.7) with

$$
\begin{aligned}
\mathbb{S}_{K, L} & :=-\frac{\alpha_{K, \sigma} \alpha_{L, \sigma}}{\alpha_{K, \sigma}+\alpha_{L, \sigma}}, \\
\mathbb{S}_{K, K} & :=\sum_{\sigma \in \mathcal{E}_{K}^{\text {int }}} \frac{\alpha_{K, \sigma} \alpha_{L, \sigma}}{\alpha_{K, \sigma}+\alpha_{L, \sigma}}+\sum_{\sigma \in \mathcal{E}_{h}^{\text {ext }} \cap \mathcal{E}_{K}} \alpha_{K, \sigma}, \\
H_{K} & :=\sum_{\sigma \in \mathcal{E}_{K}^{\text {int }}} \frac{\alpha_{K, \sigma}}{\alpha_{K, \sigma}+\alpha_{L, \sigma}}\left(\left(g, \psi_{\sigma}\right)_{K}+\left(g, \psi_{\sigma}\right)_{L}\right) .
\end{aligned}
$$

As observed in [59, 20, 57], for $g=0,(4.28 \mathrm{a})-(4.28 \mathrm{c})$ coincides with the standard two-point FV scheme $[29,31]$. The final matrix $\mathbb{S}$ is symmetric and has a $(d+2)$-point stencil, that is, there are at most $d+2$ nonzero entries per each row of $\mathbb{S}$. Let $d=2$ and $\left.\underline{\mathbf{S}}\right|_{K}=\mathbb{I} s_{K}$. Then $\mathbb{S}$ is positive definite on Delaunay meshes (a Delaunay mesh is such that the closure of the circumcircle of each simplex does not contain any other simplex vertex) but indefinite otherwise [20]. A critical situation arises, as outlined in Section 4.1.1, when two triangles cut a square, see Figure 4, left part. The local matrices $\mathbb{M}_{V}$ degenerate and the final problem (4.27) is not well-posed. In order to proceed, an "aggregation" of the two triangles into a square has to be done, leading to one final unknown for each aggregated pair of triangles. We refer for details to $[59,20,57]$. This approach is called in Section 5 below the FV method.

In the approach of Section 4.1.2, the final matrix $\mathbb{S}$ is in general nonsymmetric and has a wider stencil (for each $K \in \mathcal{T}_{h}$, all simplices sharing a node with $K$ are involved). The family of meshes where $\mathbb{S}$ is positive definite is, however, larger in comparison with the previous case. Recall that, similarly as in the previous case, singular local matrices $\mathbb{M}_{V}$ can appear, see Section 4.1.2. We refer for details to [53]. This approach is called in Section 5 below the CMFE method.

\subsubsection{Using the potential relation}

Alternatively, we can insert (4.25) into

$$
\mathbb{N} \Lambda=\bar{P},
$$

where $\mathbb{N}$ is the $\left|\mathcal{T}_{h}\right| \times\left|\mathcal{E}_{h}^{\text {int }}\right|$ matrix with rows formed by the element matrices $\mathbb{N}_{K}$ of (4.15). This gives

$$
\mathbb{N M}^{\text {inv }} \bar{P}+\bar{P}=\mathbb{N M}^{\text {inv }} \widetilde{G},
$$

i.e., (1.7) with the square matrix $\mathbb{S}:=\mathbb{N} \mathbb{M}^{\text {inv }}+\mathbb{I}$ and right-hand side vector $H:=\mathbb{N}^{\text {Minv }} \widetilde{G}$. Remark that here, (4.29) is used repeatedly. This approach is studied in detail in [55].

Let $d=2$, fix $K \in \mathcal{T}_{h}$, and consider the approach of Section 4.1.1. Recall (4.10), the nota- 


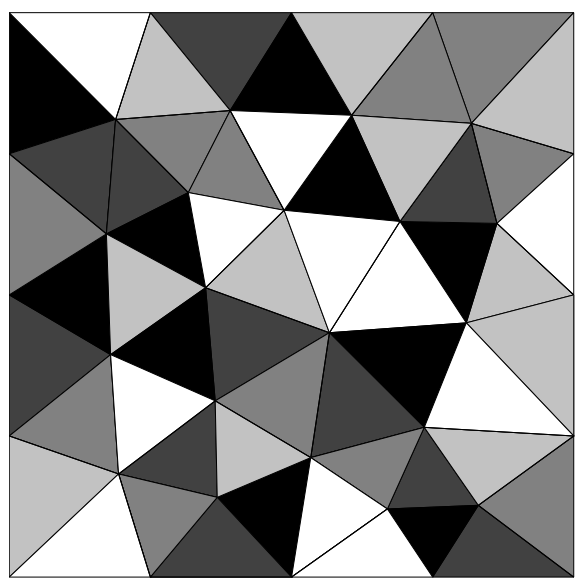

Figure 5: Initial mesh; the coloring indicates the permeability tensor $\underline{\mathbf{S}}$ in cases (5.2) and (5.3)

tion (4.11), and plug (4.12) into (4.1). This gives (1.7) with

$$
\begin{aligned}
\mathbb{S}_{K, L} & :=-\frac{\psi_{\sigma}\left(\mathbf{z}_{K}\right) \alpha_{L, \sigma}}{\alpha_{K, \sigma}+\alpha_{L, \sigma}} \\
\mathbb{S}_{K, K} & :=1-\sum_{\sigma \in \mathcal{E}_{K}^{\text {int }}} \frac{\psi_{\sigma}\left(\mathbf{z}_{K}\right) \alpha_{K, \sigma}}{\alpha_{K, \sigma}+\alpha_{L, \sigma}}, \\
H_{K} & :=\sum_{\sigma \in \mathcal{E}_{K}^{\text {int }}} \frac{\psi_{\sigma}\left(\mathbf{z}_{K}\right)}{\alpha_{K, \sigma}+\alpha_{L, \sigma}}\left(\left(g, \psi_{\sigma}\right)_{K}+\left(g, \psi_{\sigma}\right)_{L}\right) .
\end{aligned}
$$

The matrix $\mathbb{S}$ still has a $(d+2)$-point stencil and its positive definiteness and well-posedness depend on the mesh $\mathcal{T}_{h}$ and the diffusion tensor $\underline{\mathbf{S}}$ similarly as in Section 4.4.1. Likewise, the weights $w_{V, \sigma}$ of Section 4.3 have no influence in this case and can be chosen arbitrarily. In contrast to Section 4.4.1, however, $\mathbb{S}$ is in general nonsymmetric. $\mathbb{S}$ becomes symmetric if the mesh is "symmetric", consisting of the elements with the same shape, and for $\underline{\mathbf{S}}=\mathbb{I}$, as the numerical experiments in [55] indicate. Then it coincides with that of Section 4.4.1. This approach is called in Section 5 below the MFEC method.

The other approaches still lead to a nonsymmetric matrix as in Section 4.4.1. The stencil involves all simplices sharing a node with a given $K \in \mathcal{T}_{h}$. Positive definiteness and well-posedness depend again on the mesh $\mathcal{T}_{h}$ and the diffusion tensor $\underline{\mathbf{S}}$. The approaches of Sections 4.1.2 and 4.1.3, respectively, are called the MFEB and MFEO methods in Section 5 below.

\section{$5 \quad$ Numerical experiments}

The goal of this section is to carry out a comparative numerical study of the different one-unknownper-element reformulations of the RT0 MFE method, as presented in Section 4. We study the behavior of the different approaches for the homogeneous and isotropic diffusion tensor in Section 5.1, for an anisotropic diffusion tensor in Section 5.2, and for an inhomogeneous diffusion tensor in Section 5.3.

We consider the problem (1.1a) on $\Omega=(0,1) \times(0,1)$, with inhomogeneous Dirichlet boundary condition given by the function $p(x, y)=0.1 y+0.9$ instead of $(1.1 b)$. We perform the calculations on uniform refinements of the mesh viewed in Figure 5. This mesh is Delaunay, with the minimal 
and maximal angles equal to 35.4 and 88.7 degrees, respectively. A sink term $g=-0.001$ is prescribed on two elements of the initial mesh. We consider the tensor $\underline{\mathbf{S}}$ in the form

$$
\left.\underline{\mathbf{S}}\right|_{K}=\left(\begin{array}{cc}
\cos \left(\theta_{K}\right) & -\sin \left(\theta_{K}\right) \\
\sin \left(\theta_{K}\right) & \cos \left(\theta_{K}\right)
\end{array}\right)\left(\begin{array}{cc}
s_{K} & 0 \\
0 & \nu s_{K}
\end{array}\right)\left(\begin{array}{cc}
\cos \left(\theta_{K}\right) & \sin \left(\theta_{K}\right) \\
-\sin \left(\theta_{K}\right) & \cos \left(\theta_{K}\right)
\end{array}\right) \text { for } K \in \mathcal{T}_{h},
$$

where we distinguish the following three different forms:

$$
s_{K}=1 \quad \forall K \in \mathcal{T}_{h}, \quad \nu=1,
$$

i.e., the homogeneous isotropic case $(\underline{\mathbf{S}}=\mathbb{I})$, or

$$
s_{K}=1 \quad \forall K \in \mathcal{T}_{h}, \quad \theta_{K} \in\left\{\frac{\pi}{5}, \frac{3 \pi}{4}, \frac{\pi}{2}, \frac{3 \pi}{5}, \frac{\pi}{3}\right\}, \quad \nu=0.2,
$$

i.e., the homogeneous, with respect to $s_{K}$, but anisotropic case ( $\underline{\mathbf{S}}$ is a full-matrix tensor), or

$$
s_{K} \in\{10,1,0.1,0.01,0.001\}, \quad \nu=1,
$$

i.e., the inhomogeneous isotropic case ( $\underline{\mathbf{S}}$ is a varying multiple of the identity matrix). The different grey shades in Figure 5 correspond to the different choices $\theta_{K}$ and $s_{K}$ in (5.2) and (5.3), respectively. All the computations were performed in double precision on a notebook with Intel Core2 Duo 2.6 $\mathrm{GHz}$ processor and MS Windows Vista operating system. Machine precision was in the power of 1e-16. All the linear system solutions were done with the help of MATLAB 7.0.4.

We test the following methods, which are all equivalent implementations of the RT0 MFE method (1.2a)-(1.2b):

1. MFEB: the final system is of the form (1.7), imposed through (4.30), with the barycenter as the evaluation point, cf. Section 4.1.2;

2. MFEC: the final system is of the form (1.7), imposed through (4.30), with the $\underline{\mathbf{S}}$-circumcenter as the evaluation point, cf. Section 4.1.1 (the problem is given by (4.31a)-(4.31c));

3. MFEO: the final system is of the form (1.7), imposed through (4.30), while choosing the evaluation point as a function of $\mathcal{T}_{h}$ and $\underline{\mathbf{S}}$, cf. Section 4.1.3;

4. CMFE: the final system is of the form (1.7), given by the approach of [53]; considering the discrete unknowns $\bar{P}_{K}$ of (4.1) with the barycenter as the evaluation point, cf. Section 4.1.2, the system matrix $\mathbb{S}$ coincides with that of (1.7) imposed through (4.27);

5. FV: the final system is of the form (1.7), imposed through (4.27), with the $\underline{\mathbf{S}}$-circumcenter as the evaluation point, cf. Section 4.1.1 (the problem is given by (4.28a)-(4.28c)); this is the approach of $[59,20,57]$ and corresponds to the two-point FV method;

6. NCFE: the final system is of the form (3.3), imposed through (3.5), i.e., through the CR NCFE method, see Section 3.3.

We show in Figure 6 the sparsity patterns of the original MFE method (1.3) and of its equivalent reformulations of the above list, for the case of the mesh of Figure 5. Recall that the matrix sizes are respectively the number of mesh elements plus mesh faces, the number of mesh interior faces, and the number of mesh elements. In Tables $2-5$, we present various properties of the final matrix systems arising from the different equivalent reformulations. We summarize in Table 1 the different 

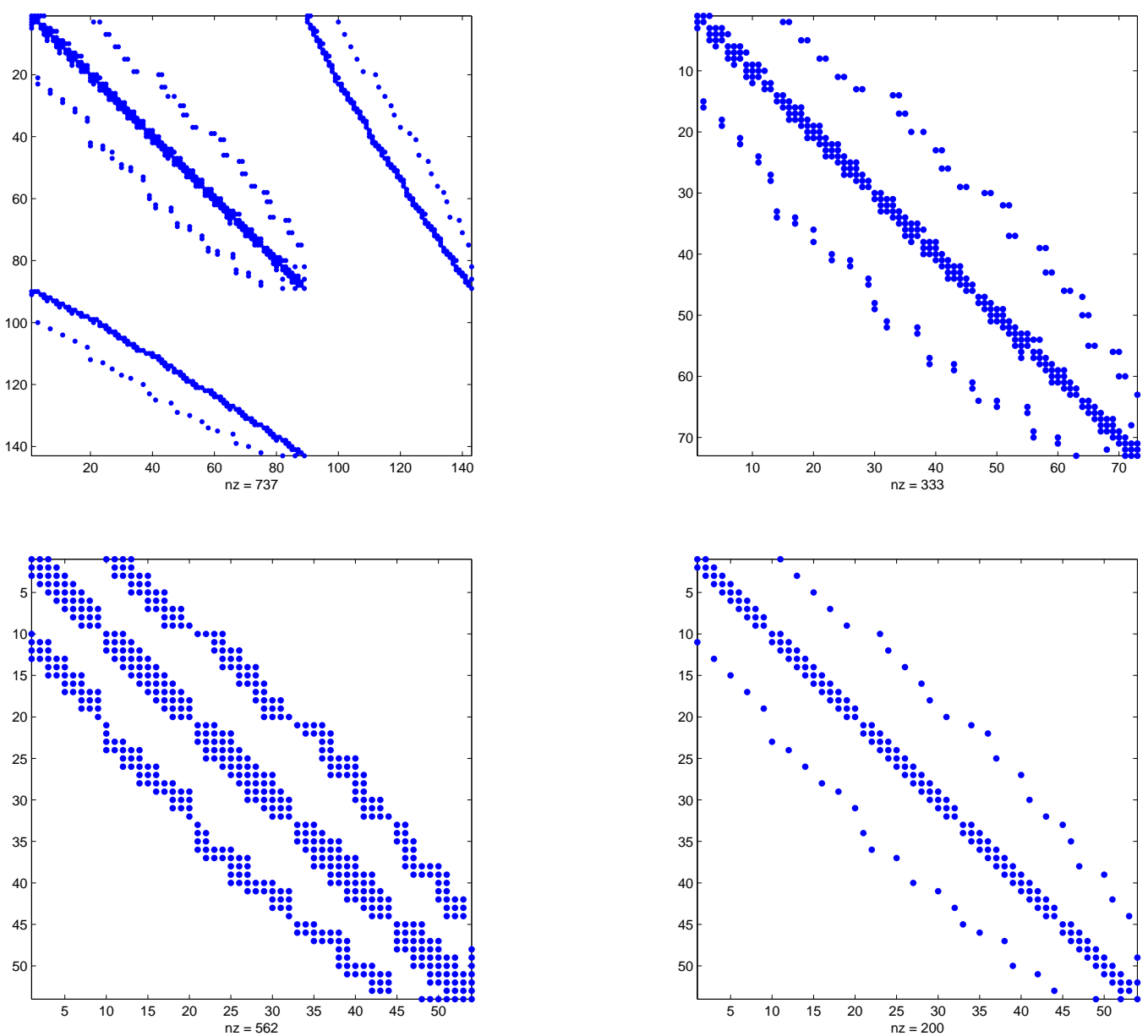

Figure 6: System matrix sparsity pattern of the original MFE formulation (top left), NCFE formulation (top right), MFEB, MFEO, and CMFE formulations (bottom left), and FV and MFEC formulations (bottom right) for the mesh of Figure 5

abbreviations used in these tables. Recall that a real matrix $\mathbb{S} \in \mathbb{R}^{M \times M}$ is positive definite if $P^{t} \mathbb{S} P>0$ for all $P \in \mathbb{R}^{M}, P \neq 0$, and negative stable when all its eigenvalues have positive real parts (this is in particular the case for positive definite matrices). The 2-norm condition number of a matrix $\mathbb{S}$ is defined by $\|\mathbb{S}\|_{2}\left\|\mathbb{S}^{-1}\right\|_{2}$. We also consider the 2-norm condition number after diagonal scaling, by which we mean the minimal of the two 2-norm condition numbers of the two matrices

$$
(\operatorname{diag}(\mathbb{S}))^{-1} \mathbb{S}, \quad|\operatorname{diag}(\mathbb{S})|^{-1 / 2} \mathbb{S}|\operatorname{diag}(\mathbb{S})|^{-1 / 2}
$$

We also study the computational cost. To do so, we restrict ourselves to standard Matlab routines and test direct and iterative solvers. We first test the \direct solver. Such a solver may not be usable for very large systems or may not be suitable for parabolic or nonlinear problems. Thus the behavior of iterative solvers is also very important. We test two iterative methods. If the matrix is symmetric and positive definite, we use the conjugate gradients method [36]. For nonsymmetric matrices, we employ the bi-conjugate gradients stabilized method [52]. Unpreconditioned iterative linear solvers may be rather slow but usually illustrate well the matrix properties and especially the matrix condition number. To accelerate their convergence, we use incomplete Cholesky and 


\begin{tabular}{ll}
\hline Abbreviation & Meaning \\
\hline Meth. & method, one of the equivalent MFE formulations \\
Un. & number of unknowns (matrix size) \\
Mat. & matrix \\
St. & stencil (the maximum number of nonzero entries on each matrix row) \\
Nonz. & total number of matrix nonzero entries \\
CN & 2-norm condition number \\
CNS & 2-norm condition number after diagonal scaling \\
DS & direct linear solver \\
CG & conjugate gradients iterative linear solver \\
PCG & preconditioned conjugate gradients iterative linear solver \\
Bi-CGStab & bi-conjugate gradients stabilized iterative linear solver \\
PBi-CGStab & preconditioned bi-conjugate gradients stabilized iterative linear solver \\
CPU & CPU time of a direct/iterative linear solver \\
Iter. & number of iterations of an iterative linear solver \\
IC & CPU time of incomplete Cholesky factorization with a specified drop tolerance \\
ILU & CPU time of incomplete LU factorization with a specified drop tolerance \\
SPD & symmetric positive definite \\
SID & symmetric indefinite \\
NPD & nonsymmetric positive definite \\
NNS & nonsymmetric negative stable \\
NID & nonsymmetric indefinite \\
\hline
\end{tabular}

Table 1: Abbreviations used in Tables 2-5

incomplete LU factorizations with a specified drop tolerance, cf. [49]. The drop tolerance is always chosen in such a way that the sum of CPU times of the preconditioning and of the solution of the preconditioned system was minimal. We always use a zero start vector and stop the iterative process as soon as the relative residual $\|H-\mathbb{S} \tilde{P}\|_{2} /\|H\|_{2}$, where $\tilde{P}$ is the approximate solution to the system $\mathbb{S} P=H$, decreases below 1e-8.

Some general conclusions may be drawn from the theoretical investigations and from Tables 2-5. NCFE always produces a symmetric positive definite matrix. The FV matrix is always symmetric, but positive definiteness depends on the tensor $\underline{\mathbf{S}}$ and on the mesh $\mathcal{T}_{h}$. All the other methods lead to nonsymmetric matrices. These matrices can be positive definite, negative stable, or indefinite, depending on $\underline{\mathbf{S}}$ and $\mathcal{T}_{h}$. FV and MFEC produce four nonzero entries per matrix row in the interior of the mesh. In the NCFE case, this number is equal to 5 . The stencil of the other methods is variable; on the row associated with a given element $K$, there are nonzero entries on columns associated with those elements $L$ which share a vertex with $K$. In the present case, this is equal to 14 in the interior of the mesh. MFEC and FV lead to the fewest total nonzero matrix entries; NCFE has roughly twice and the other methods have roughly three times as many nonzeros.

\subsection{Identity matrix diffusion tensor}

Tables 2 and 3 present the results for the coefficients (5.1) and respectively fourth- and fifth-level uniform refinements of the mesh of Figure 5. The condition number of all methods is here roughly comparable; the only (negative) exception is FV. The condition numbers get mutually much closer 


\begin{tabular}{|c|c|c|c|c|c|c|c|c|c|c|c|c|}
\hline \multirow[b]{2}{*}{ Meth. } & \multirow[b]{2}{*}{ Un. } & \multirow[b]{2}{*}{ Mat. } & \multirow[b]{2}{*}{ St. } & \multirow[b]{2}{*}{ Nonz. } & \multirow[b]{2}{*}{$\mathrm{CN}$} & \multirow[b]{2}{*}{ CNS } & \multirow{2}{*}{$\begin{array}{r}\text { DS } \\
\text { CPU }\end{array}$} & \multicolumn{2}{|c|}{$\begin{array}{c}\text { CG/ } \\
\text { Bi-CGStab }\end{array}$} & \multicolumn{3}{|c|}{$\begin{array}{c}\text { PCG/ } \\
\text { PBi-CGStab }\end{array}$} \\
\hline & & & & & & & & $\mathrm{CPU}$ & Iter. & $\mathrm{CPU}$ & $\begin{array}{l}\text { IC/ } \\
\text { ILU }\end{array}$ & Iter. \\
\hline MFEB & 13824 & NPD & 14 & 177652 & 7564 & 7580 & 0.27 & 4.86 & 324.5 & 0.81 & 0.36 & 9.0 \\
\hline MFEC & 13824 & NNS & 4 & 55040 & 11256 & 11056 & 0.09 & 2.23 & 372.0 & 0.42 & 0.19 & 6.5 \\
\hline MFEO & 13824 & NPD & 14 & 177652 & 7531 & 7558 & 0.28 & 4.08 & 270.0 & 0.80 & 0.41 & 7.5 \\
\hline CMFE & 13824 & NPD & 14 & 177652 & 7397 & 7380 & 0.27 & 4.70 & 312.0 & 0.83 & 0.39 & 8.5 \\
\hline $\mathrm{FV}$ & 13824 & SPD & 4 & 55040 & 65722 & 8898 & 0.07 & 3.09 & 1098.0 & 0.42 & 0.17 & 17.0 \\
\hline NCFE & 20608 & SPD & 5 & 102528 & 14064 & 9944 & 0.14 & 2.92 & 620.0 & 1.11 & 0.56 & 19.0 \\
\hline
\end{tabular}

Table 2: Matrix properties and computational cost of the different equivalent formulations of the MFE method, identity matrix diffusion tensor (5.1), fourth-level mesh refinement

\begin{tabular}{|c|c|c|c|c|c|c|c|c|c|c|c|c|}
\hline \multirow[b]{2}{*}{ Meth. } & \multirow[b]{2}{*}{ Un. } & \multirow[b]{2}{*}{ Mat. } & \multirow[b]{2}{*}{ St. } & \multirow[b]{2}{*}{ Nonz. } & \multirow[b]{2}{*}{$\mathrm{CN}$} & \multirow[b]{2}{*}{ CNS } & \multirow{2}{*}{$\begin{array}{r}\text { DS } \\
\text { CPU }\end{array}$} & \multicolumn{2}{|c|}{$\begin{array}{c}\text { CG/ } \\
\text { Bi-CGStab }\end{array}$} & \multicolumn{3}{|c|}{$\begin{array}{c}\text { PCG/ } \\
\text { PBi-CGStab }\end{array}$} \\
\hline & & & & & & & & $\mathrm{CPU}$ & Iter. & $\mathrm{CPU}$ & $\begin{array}{l}\mathrm{IC} / \\
\mathrm{ILU}\end{array}$ & Iter. \\
\hline MFEB & 55296 & NPD & 14 & 714740 & 30289 & 30373 & 1.51 & 33.31 & 530.0 & 8.14 & 3.37 & 19.5 \\
\hline MFEC & 55296 & NNS & 4 & 220672 & 45016 & 44643 & 0.49 & 17.47 & 624.5 & 3.34 & 1.25 & 13.5 \\
\hline MFEO & 55296 & NPD & 14 & 714740 & 30143 & 30285 & 1.55 & 37.76 & 594.5 & 7.06 & 2.75 & 19.5 \\
\hline CMFE & 55296 & NPD & 14 & 714740 & 29630 & 29551 & 1.49 & 34.32 & 544.5 & 6.91 & 2.73 & 18.5 \\
\hline $\mathrm{FV}$ & 55296 & SPD & 4 & 220672 & 263036 & 35813 & 0.48 & 29.52 & 2170.0 & 3.08 & 1.47 & 20.0 \\
\hline NCFE & 82688 & SPD & 5 & 412416 & 56416 & 39901 & 0.90 & 35.80 & 1219.0 & 9.41 & 3.78 & 42.0 \\
\hline
\end{tabular}

Table 3: Matrix properties and computational cost of the different equivalent formulations of the MFE method, identity matrix diffusion tensor (5.1), fifth-level mesh refinement

after the diagonal scaling. The matrix size and sparsity pattern/number of nonzero entries imply that FV/MFEC give smallest CPU times while using the direct solver, followed by NCFE and then all other methods. MFEC behaves best for an unpreconditioned linear solver, actually much better than FV which do has the advantage of a symmetric matrix. MFEB/MFEC/MFEO proposed in the present paper and the related CMFE seem to outperform NCFE for increasing mesh size (a systematically better behavior of CMFE over NCFE is observed in [53]). Concerning preconditioned iterative solvers, FV/MFEC seem to perform roughly two times as fast as MFEB/MFEO/CMFE and roughly three times as fast as NCFE.

\subsection{Anisotropic diffusion tensor}

Table 4 presents the results for the coefficients (5.2) and the fourth-level uniform refinement of the mesh of Figure 5. Because of the anisotropy of the diffusion tensor, FV leads to a symmetric indefinite matrix, whereas MFEC to a nonsymmetric indefinite matrix. These matrices are also very badly conditioned, whereby the diagonal scaling does not help too much. Consequently, direct application of the iterative solvers leads to no convergence in 50000 iterations. All the other 


\begin{tabular}{|c|c|c|c|c|c|c|c|c|c|c|c|c|}
\hline \multirow[b]{2}{*}{ Meth. } & \multirow[b]{2}{*}{ Un. } & \multirow[b]{2}{*}{ Mat. } & \multirow[b]{2}{*}{ St. } & \multirow[b]{2}{*}{ Nonz. } & \multirow[b]{2}{*}{$\mathrm{CN}$} & \multirow[b]{2}{*}{ CNS } & \multirow{2}{*}{$\begin{array}{r}\text { DS } \\
\text { CPU }\end{array}$} & \multicolumn{2}{|c|}{$\begin{array}{c}\text { CG/ } \\
\text { Bi-CGStab } \\
\end{array}$} & \multicolumn{3}{|c|}{$\begin{array}{c}\text { PCG/ } \\
\text { PBi-CGStab }\end{array}$} \\
\hline & & & & & & & & $\mathrm{CPU}$ & Iter. & $\mathrm{CPU}$ & $\begin{array}{l}\text { IC/ } \\
\text { ILU }\end{array}$ & Iter. \\
\hline MFEB & 13824 & NPD & 14 & 177652 & 14489 & 11203 & 0.28 & 6.61 & 448.0 & 0.98 & 0.59 & 6.5 \\
\hline MFEC & 13824 & NID & 4 & 55040 & 2401279 & 416769 & 0.08 & - & - & 0.45 & 0.20 & 7.0 \\
\hline MFEO & 13824 & NPD & 14 & 177652 & 13401 & 10767 & 0.27 & 6.51 & 440.5 & 0.95 & 0.41 & 10.0 \\
\hline CMFE & 13824 & NPD & 14 & 177652 & 9276 & 7758 & 0.28 & 5.27 & 350.5 & 0.84 & 0.38 & 9.0 \\
\hline $\mathrm{FV}$ & 13824 & SID & 4 & 55040 & 247055 & 239934 & 0.09 & - & - & 0.45 & 0.20 & 7.0 \\
\hline NCFE & 20608 & SPD & 5 & 102528 & 25393 & 16969 & 0.18 & 4.03 & 850.0 & 1.12 & 0.41 & 30.0 \\
\hline
\end{tabular}

Table 4: Matrix properties and computational cost of the different equivalent formulations of the MFE method, anisotropic diffusion tensor (5.2), fourth-level mesh refinement

\begin{tabular}{|c|c|c|c|c|c|c|c|c|c|c|c|c|}
\hline \multirow[b]{2}{*}{ Meth. } & \multirow[b]{2}{*}{ Un. } & \multirow[b]{2}{*}{ Mat. } & \multirow[b]{2}{*}{ St. } & \multirow[b]{2}{*}{ Nonz. } & \multirow[b]{2}{*}{$\mathrm{CN}$} & \multirow[b]{2}{*}{ CNS } & \multirow{2}{*}{$\begin{array}{r}\text { DS } \\
\text { CPU }\end{array}$} & \multicolumn{2}{|c|}{$\begin{array}{c}\text { CG/ } \\
\text { Bi-CGStab }\end{array}$} & \multicolumn{3}{|c|}{$\begin{array}{c}\text { PCG/ } \\
\text { PBi-CGStab }\end{array}$} \\
\hline & & & & & & & & $\mathrm{CPU}$ & Iter. & $\mathrm{CPU}$ & $\begin{array}{l}\text { IC/ } \\
\text { ILU }\end{array}$ & Iter. \\
\hline MFEB & 13824 & NPD & 14 & 177652 & 819248 & 740706 & 0.28 & 13.33 & 897.5 & 1.05 & 0.62 & 6.5 \\
\hline MFEC & 13824 & NNS & 4 & 55040 & 903789 & 763849 & 0.09 & 5.34 & 947.5 & 0.47 & 0.20 & 7.5 \\
\hline MFEO & 13824 & NPD & 14 & 177652 & 820367 & 739957 & 0.28 & 12.45 & 790.5 & 1.05 & 0.56 & 8.0 \\
\hline CMFE & 13824 & NPD & 14 & 177652 & 2500730 & 478974 & 0.28 & 102.27 & 6842.5 & 1.01 & 0.41 & 10.5 \\
\hline $\mathrm{FV}$ & 13824 & SPD & 4 & 55040 & 16387758 & 497974 & 0.07 & 39.41 & 14101.0 & 0.44 & 0.17 & 16.0 \\
\hline NCFE & 20608 & SPD & 5 & 102528 & 4797335 & 670623 & 0.18 & 52.42 & 11226.0 & 1.22 & 0.64 & 16.0 \\
\hline
\end{tabular}

Table 5: Matrix properties and computational cost of the different equivalent formulations of the MFE method, inhomogeneous diffusion tensor (5.3), fourth-level mesh refinement

methods behave rather similarly to Table 2. Application of the direct solver or of iterative solvers with preconditioning leads to results similar to that of Table 2 .

\subsection{Inhomogeneous diffusion tensor}

Table 5 presents the results for the coefficients (5.3) and the fourth-level uniform refinement of the mesh of Figure 5. Here FV gives a symmetric positive definite matrix and MFEC a nonsymmetric negative stable matrix. The inhomogeneity of the diffusion tensor however causes an increase of the matrices condition numbers. This increase is severe in CMFE and NCFE, and in particular in FV. Consequently, direct application of the iterative solvers leads to important increase of the CPU time in CMFE, NCFE, and FV. Whereas FV and MFEC behaved similarly in Table 2, MFEC becomes here almost 8 times faster than the FV one. When diagonal scaling is applied, however, the condition numbers of all methods become comparable, whence the preconditioned iterative solvers behave similarly as for the case of Table 2. Also the application of the direct solver leads to results similar to those of Table 2 . 


\subsection{Discussion}

Amongst the above-tested equivalent formulations, the MFEC leads to very compact 4-point stencils in two space dimensions and excellent computational performance for problems with a possibly highly inhomogeneous diffusion tensor. The FV has similar properties and leads, in addition, to symmetric matrices, but gives increased matrix condition numbers for highly inhomogeneous diffusion tensors. Both reformulations, however, seem to behave less well for anisotropic diffusion tensors, and, moreover, are only applicable in two space dimensions. Then the MFEB, MFEO, or the previously proposed CMFE reformulations seem to be appealing alternatives to the classical NCFE implementation.

\section{The discrete maximum principle}

We recall in this section the different variants of the discrete maximum principle valid for the RT0 MFE method. For this purpose, we replace the homogeneous Dirichlet boundary condition (1.1b) by a nonhomogeneous one,

$$
p=f \quad \text { on } \partial \Omega,
$$

with $f \in H^{\frac{1}{2}}(\partial \Omega)$.

Recall from Section 3.3 the notation $\psi_{\sigma}$ for the CR basis function of a side $\sigma \in \mathcal{E}_{h}$ and the equivalence of the lowest-order mixed and nonconforming elements. Then we have the following classical result; in a more general setting, it has been shown in, e.g., [34, Theorem 4.5]:

Theorem 6.1 (Discrete maximum principle for the Lagrange multipliers $\lambda_{h}$ ). Let

$$
\left(\underline{\mathbf{S}} \nabla \psi_{\gamma}, \nabla \psi_{\sigma}\right) \leq 0
$$

for all sides $\sigma \in \mathcal{E}_{h}^{\text {int }}$ and all $\gamma \in \mathcal{E}_{h}$ such that $\sigma$ and $\gamma$ lie in the same simplex $K \in \mathcal{T}_{h}$. Let $g \geq 0$ and $f \geq 0$. Then $\left.\lambda_{h}\right|_{\sigma}=\Lambda_{\sigma} \geq 0$ for all $\sigma \in \mathcal{E}_{h}$.

Let $\underline{\mathbf{S}}$ be such that it can be written as $\mathbb{I}$ times a piecewise constant scalar function and let $\mathcal{T}_{h}$ be weakly acute, i.e., such that the magnitude of the angles between $\mathbf{n}_{K, \sigma}, \sigma \in \mathcal{E}_{K}$, for all $K \in \mathcal{T}_{h}$ is greater than or equal to $\pi / 2$ (all angles smaller than or equal to $\pi / 2$ in two space dimensions). Then (6.1) holds. Thus, the discrete maximum principle holds for the Lagrange multipliers $\lambda_{h}$ of (3.1a)-(3.1c) under this condition. Note that one does not necessarily have the discrete maximum principle for the function $\lambda_{h}^{\mathrm{NC}}$ defined in (3.6), as this function may take values larger than $\max _{\sigma \in \mathcal{E}_{h}} \Lambda_{\sigma}$ and smaller than $\min _{\sigma \in \mathcal{E}_{h}} \Lambda_{\sigma}$.

Note that by properties of a simplex and by the fact that $\lambda_{h}^{\mathrm{NC}}$ is an affine function,

$$
\lambda_{h}^{\mathrm{NC}}\left(\mathbf{x}_{K}\right)=\frac{1}{d+1} \sum_{\sigma \in \mathcal{E}_{K}} \Lambda_{\sigma}
$$

for all $K \in \mathcal{T}_{h}$. Recalling the formula (3.7b) relating $\lambda_{h}^{\mathrm{NC}}$ and $p_{h}$, the discrete maximum principle also holds for the original piecewise constant approximation $p_{h}$ :

Theorem 6.2 (Discrete maximum principle for $p_{h}$ ). Let the assumptions of Theorem 6.1 be satisfied. Then $p_{h} \geq 0$.

Finally, we will employ the link between the RT0 MFE and the two-point FV from Section 4.4.1. From the form of the final system (1.7) given in this case by (4.28a)-(4.28c), we have: 


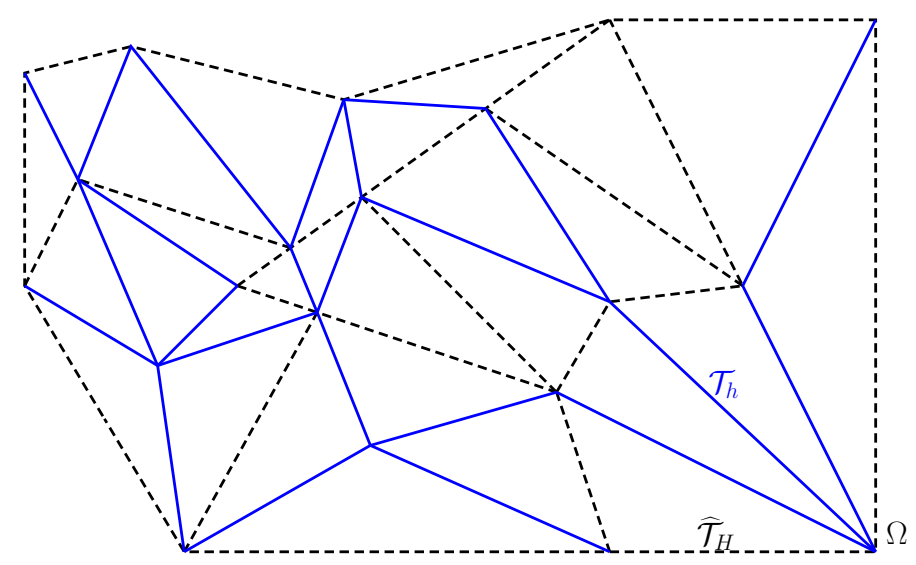

Figure 7: Example of a general polygonal mesh $\widehat{\mathcal{T}}_{H}$ (dashed lines) and of a possible simplicial submesh $\mathcal{T}_{h}$ (solid lines)

Theorem 6.3 (Discrete maximum principle for the circumcenter values of $\lambda_{h}^{\mathrm{NC}}$ ). Let $d=2$ and

$$
\begin{aligned}
\frac{\alpha_{K, \sigma} \alpha_{L, \sigma}}{\alpha_{K, \sigma}+\alpha_{L, \sigma}} \geq 0 & \forall \sigma \in \mathcal{E}_{h}^{\text {int }}, \\
\alpha_{K, \sigma} \geq 0 & \forall \sigma \in \mathcal{E}_{h}^{\text {ext }} .
\end{aligned}
$$

Let next $g \geq 0, f \geq 0$, and

$$
H_{K} \geq 0 \quad \forall K \in \mathcal{T}_{h},
$$

where $H$ is the right-hand side vector from (4.28c). Then $\left.\lambda_{h}^{\mathrm{NC}}\right|_{K}\left(\mathbf{z}_{K}\right)=\bar{P}_{K} \geq 0$ for all $K \in \mathcal{T}_{h}$.

Let $d=2$, let $\underline{\mathbf{S}}=\mathbb{I}$ for simplicity, let $\mathcal{T}_{h}$ be Delaunay, and let finally each circumcenter of a boundary triangle be inside $\Omega$. Then (6.2a)-(6.2b) holds, cf. [20]. (Extensions to triangulations obtained from square meshes are also possible following [20]). The requirement (6.3) of course holds when $g=0$ (when the RT0 MFE and the two-point FV coincide), but otherwise, (6.3) adds an additional restriction on the mesh and the diffusion-dispersion tensor $\underline{\mathbf{S}}$. In any case, under the hypotheses of Theorem 6.3, one also has the discrete maximum principle for the values of $\lambda_{h}^{\mathrm{NC}}$ in the circumcenters of the elements of $\mathcal{T}_{h}$.

\section{General polygonal meshes and relations to other methods}

The aim of this section is to recall that MFEs (of arbitrary order) can be used on general polygonal meshes and to study their relations to other locally conservative methods.

Let $\widehat{\mathcal{T}}_{H}$ be a mesh consisting of general polygonal elements. We give an example in Figure 7 (dashed lines). The mesh $\widehat{\mathcal{T}}_{H}$ does not need to be matching (can contain hanging nodes), the maximal number of sides of each element $K \in \widehat{\mathcal{T}}_{H}$ is not necessarily limited, $\widehat{\mathcal{T}}_{H}$ is not necessarily shape-regular, and its elements can be nonconvex and non star-shaped. We only need the existence of a simplicial submesh $\mathcal{T}_{h}$ of $\widehat{\mathcal{T}}_{H}$ (that is, every element of $\widehat{\mathcal{T}}_{H}$ is triangulated by elements of $\mathcal{T}_{h}$ ) which is matching. An example is given in Figure 7 (solid lines). We will show that MFEs of arbitrary order can be defined on such polygonal meshes and written with potential unknowns only related to the elements of $\widehat{\mathcal{T}}_{H}$ and fluxes/Lagrange multipliers only related to the sides of $\widehat{\mathcal{T}}_{H}$. For this purpose, we start either from (1.3) or from (3.3). Recall from [16, 47] that any order MFE method from any of the different families on a simplicial mesh $\mathcal{T}_{h}$ can be written under this form. 
Theorem 7.1 (Mixed finite elements on polygonal meshes for Lagrange multipliers). Let $\widehat{\mathcal{T}}_{H}$ be an arbitrary polygonal mesh with a matching simplicial submesh $\mathcal{T}_{h}$. Consider any MFE scheme on $\mathcal{T}_{h}$, written for the Lagrange multipliers $\Lambda$ associated with the sides of $\mathcal{T}_{h}$ as

$$
\mathbb{Z} \Lambda=E
$$

Then, (7.1) can be condensed into

$$
\widehat{\mathbb{Z}} \widehat{\Lambda}=\widehat{E}
$$

with a sparse, symmetric, and positive definite matrix $\widehat{\mathbb{Z}} . \widehat{\Lambda}$ is an algebraic vector of Lagrange multipliers related to the sides of $\widehat{\mathcal{T}}_{H}$ only. In the RTO case (3.3) and when the sides of $\mathcal{T}_{h}$ do not subdivide the sides of $\widehat{\mathcal{T}}_{H}$, there is one unknown in $\widehat{\Lambda}$ per side of $\widehat{\mathcal{T}}_{H}$.

Proof. Note first that (7.2) has the same structure as (7.1). The proof follows by a simple static condensation. Indeed, let a $K \in \widehat{\mathcal{T}}_{H}$ be given and denote the unknowns $\Lambda$ of (7.1) corresponding to the sides of $\mathcal{T}_{h}$ which are in the interior of $K$ by $\Lambda_{K}^{\text {int }}$. Use a similar notation $E_{K}^{\text {int }}$ for the right-hand side entries of (7.1). Finally, denote the unknowns corresponding to sides of $\mathcal{T}_{h}$ which are on the boundary of $K$ but not on the boundary of $\Omega$ by $\Lambda_{K}^{\text {ext }}$. Consider the lines of (7.1) associated with such sides of $\mathcal{T}_{h}$ which are in the interior of $K$. This gives rise to the following local problem: given $\Lambda_{K}^{\text {ext }}$, find $\Lambda_{K}^{\text {int }}$ such that

$$
\mathbb{Z}_{K}^{\mathrm{int}} \Lambda_{K}^{\mathrm{int}}=E_{K}^{\mathrm{int}}-\mathbb{Z}_{K}^{\mathrm{ext}} \Lambda_{K}^{\mathrm{ext}}
$$

Note that the system matrix $\mathbb{Z}_{K}^{\text {int }}$ is square, symmetric, and positive definite, which implies the wellposedness of the local problem $\left(\mathbb{Z}_{K}^{\text {int }}\right.$ is a submatrix of $\mathbb{Z}$ corresponding to the lines and columns corresponding to the sides of $\mathcal{T}_{h}$ which are in the interior of $\left.K\right)$. Note also that (7.3) is a local Dirichlet problem, which allows to compute $\Lambda_{K}^{\text {int }}$ from $\Lambda_{K}^{\text {ext }}$ and $E_{K}^{\text {int }}$. More precisely, we have

$$
\Lambda_{K}^{\mathrm{int}}=\left(\mathbb{Z}_{K}^{\mathrm{int}}\right)^{-1}\left(E_{K}^{\mathrm{int}}-\mathbb{Z}_{K}^{\mathrm{ext}} \Lambda_{K}^{\mathrm{ext}}\right) .
$$

Next, we repeat this procedure for all $K \in \widehat{\mathcal{T}}_{H}$. We finally use the lines of (7.1) associated with the sides of $\mathcal{T}_{h}$ which are on the boundary of some $K \in \widehat{\mathcal{T}}_{H}$ but not on the boundary of $\Omega$, where we insert the expressions (7.4). This gives (7.2). Note that, in contrast to Section 4, all equations of (7.1) are used exactly once; this process is called static condensation and clearly leads to the well-posedness of (7.2) with $\widehat{\mathbb{Z}}$ being sparse, symmetric, and positive definite.

Theorem 7.2 (Mixed finite elements on polygonal meshes for flux and potential unknowns). Let $\widehat{\mathcal{T}}_{H}$ be an arbitrary polygonal mesh with a matching simplicial submesh $\mathcal{T}_{h}$. Consider any MFE scheme on $\mathcal{T}_{h}$ leading to

$$
\left(\begin{array}{ll}
\mathbb{A} & \mathbb{B}^{t} \\
\mathbb{B} & 0
\end{array}\right)\left(\begin{array}{c}
U \\
P
\end{array}\right)=\left(\begin{array}{c}
F \\
G
\end{array}\right)
$$

Then, (7.5) can be condensed into a well-posed system of the form

$$
\left(\begin{array}{ll}
\widehat{\mathbb{A}} & \widehat{\mathbb{B}}^{t} \\
\widehat{\mathbb{B}} & 0
\end{array}\right)\left(\begin{array}{l}
\widehat{U} \\
\widehat{P}
\end{array}\right)=\left(\begin{array}{c}
\widehat{F} \\
\widehat{G}
\end{array}\right),
$$

where the system matrix is sparse and of indefinite, saddle point type, with $\widehat{\mathbb{A}}$ symmetric and positive definite and $\widehat{\mathbb{B}}$ of full row rank. $\widehat{U}$ is an algebraic vector of flux unknowns related to the sides of $\widehat{\mathcal{T}}_{H}$ only, and $\widehat{P}$ is an algebraic vector of potential unknowns related to the elements of $\widehat{\mathcal{T}}_{H}$ only. In the RTO case (1.3) and when the sides of $\mathcal{T}_{h}$ do not subdivide the sides of $\widehat{\mathcal{T}}_{H}$, there is one unknown in $\widehat{U}$ per side of $\widehat{\mathcal{T}}_{H}$ and one unknown in $\widehat{P}$ per element of $\widehat{\mathcal{T}}_{H}$. 
Proof. The proof follows by a simple static condensation. We present here briefly its algebraic form; more details are given below in Theorem 7.4. Note first that (7.6) has the same structure as (7.5). Consider such basis of the space $\Phi_{h}$ of (1.2a)-(1.2b) which contains the indicator functions $\phi_{K}$ of all $K \in \widehat{\mathcal{T}}_{H}$, i.e., the functions equal to one on $K$ and zero elsewhere (then, all other basis functions of $\Phi_{h}$ have zero mean value on each $K \in \widehat{\mathcal{T}}_{H}$ ). Let a $K \in \widehat{\mathcal{T}}_{H}$ be given and consider the lines of the first block of (7.5), i.e., of $\left(\mathbb{A} \mathbb{B}^{t}\right)$, associated with such sides of $\mathcal{T}_{h}$ which are in the interior of $K$. Consider moreover the lines of the second block of (7.5), i.e., of ( $\mathbb{B} 0$ ), associated with all basis functions with support in $K$, other than $\phi_{K}$. This gives rise to the following local problem: given $U_{K}^{\text {ext }}$, find $U_{K}^{\text {int }}$ and $P_{K}^{0}$ such that

$$
\left(\begin{array}{cc}
\mathbb{A}_{K}^{\text {int,int }} & \left(\mathbb{B}_{K}^{0, \text { int }}\right)^{t} \\
\mathbb{B}_{K}^{0, \text { int }} & 0
\end{array}\right)\left(\begin{array}{c}
U_{K}^{\text {int }} \\
P_{K}^{0}
\end{array}\right)=\left(\begin{array}{c}
F_{K}^{\text {int }}-\mathbb{A}_{K}^{\text {int,ext }} U_{K}^{\text {ext }} \\
G_{K}^{0}-\mathbb{B}_{K}^{0, \text { ext }} U_{K}^{\text {ext }}
\end{array}\right) .
$$

The well-posedness of this local problem follows from the fact that it corresponds to a local Neumann problem with compatible data (the compatibility of the data follows from (7.5)). Using (7.7), we can compute the fluxes $U_{K}^{\text {int }}$ and potentials $P_{K}^{0}$ in the interior of $K$ as a function of the fluxes $U_{K}^{\text {ext }}$ through the boundary of $K$, of $F_{K}^{\text {int }}$, and of $G_{K}^{0}$. Note that the matrix of (7.7) is formed by lines and columns of the matrix of (7.5). It is now sufficient to insert these expressions for all $K \in \widehat{\mathcal{T}}_{H}$ into the remaining equations of (7.5), i.e., those associated with the lines of the first block of (7.5) associated with the sides of $\mathcal{T}_{h}$ which are on the boundary of some $K \in \widehat{\mathcal{T}}_{H}$ and those associated with the lines of the second block of (7.5) associated with the basis functions $\phi_{K}$. This leads to a system of a form (7.6).

Remark 7.3 (Mixed finite element approximation approaches). In the approach of [39, 40], see also the references therein, and in [50], a matching simplicial submesh is also introduced to define the RTO MFE on polygonal meshes. However, therein, some degrees of freedom are "frozen" or linked explicitly to the other ones, which can be seen as an approximation using a numerical quadrature. Consequently, one does not obtain the same result as in (1.2a)-(1.2b), contrarily to our approach.

We finally study the relation of MFE methods (7.2) and (7.6) in the lowest-order case with some popular polygonal discretization schemes.

Theorem 7.4 (Relation with mimetic finite difference, mixed finite volume, and hybrid finite volume methods). Let the source term $g$ be piecewise constant on $\widehat{\mathcal{T}}_{H}$, let the sides of $\mathcal{T}_{h}$ do not subdivide the sides of $\widehat{\mathcal{T}}_{H}$, and consider the RTO case (1.3). Then the MFE method (7.6) belongs to the MFD family as characterized in [17]. More generally, both (7.2) and (7.6) belong to the family characterized in [27].

Proof. We first show that (7.6) belongs to the MFD family as characterized in [17]. Let $K \in \widehat{\mathcal{T}}_{H}$ be fixed. Inverting the system matrix in (7.7) gives

$$
\left(\begin{array}{c}
U_{K}^{\text {int }} \\
P_{K}^{0}
\end{array}\right)=\left(\begin{array}{cc}
\mathbb{A}_{K}^{\text {int,int }} & \left(\mathbb{B}_{K}^{0, \text { int }}\right)^{t} \\
\mathbb{B}_{K}^{0, \text { int }} & 0
\end{array}\right)^{-1}\left(\begin{array}{l}
F_{K}^{\text {int }}-\mathbb{A}_{K}^{\text {int,ext }} U_{K}^{\text {ext }} \\
G_{K}^{0}-\mathbb{B}_{K}^{0, \text { ext }} U_{K}^{\text {ext }}
\end{array}\right) .
$$

Let us denote by $P_{K}^{1}$ the coefficient associated with the $\phi_{K}$ basis function (this is the value of the vector $\widehat{P}$ associated with $K)$. The equations of (7.5) which were not used in (7.7) related to the element $K$ read

$$
\left(\begin{array}{cc}
\mathbb{A}_{K}^{\text {ext,ext }} & \left(\mathbb{A}_{K}^{\text {int,ext }}\right)^{t} \\
\mathbb{B}_{K}^{1, \text { ext }} & 0
\end{array}\right)\left(\begin{array}{c}
U_{K}^{\text {ext }} \\
U_{K}^{\text {int }}
\end{array}\right)+\left(\begin{array}{cc}
\left(\mathbb{B}_{K}^{1, \text { ext }}\right)^{t} & \left(\mathbb{B}_{K}^{0, \text { ext }}\right)^{t} \\
0 & 0
\end{array}\right)\left(\begin{array}{c}
P_{K}^{1} \\
P_{K}^{0}
\end{array}\right)=\left(\begin{array}{c}
F_{K}^{\text {ext }} \\
G_{K}^{1}
\end{array}\right) .
$$


Plugging (7.8) into (7.9) gives the following block system for the unknowns $\left(U_{K}^{\text {ext }}, P_{K}^{1}\right)$ :

$$
\left(\begin{array}{ll}
\widehat{\mathbb{A}}_{K} & \left(\mathbb{B}_{K}^{1, \text { ext }}\right)^{t} \\
\mathbb{B}_{K}^{1, \text { ext }} & 0
\end{array}\right)\left(\begin{array}{c}
U_{K}^{\text {ext }} \\
P_{K}^{1}
\end{array}\right)=\left(\begin{array}{c}
\widehat{F}_{K}^{\text {ext }} \\
G_{K}^{1}
\end{array}\right),
$$

where

$$
\widehat{\mathbb{A}}_{K}:=\mathbb{A}_{K}^{\text {ext,ext }}-\left(\begin{array}{l}
\mathbb{A}_{K}^{\text {int,ext }} \\
\mathbb{B}_{K}^{0, \text { ext }}
\end{array}\right)^{t}\left(\begin{array}{cc}
\mathbb{A}_{K}^{\text {int,int }} & \left(\mathbb{B}_{K}^{0, \text { int }}\right)^{t} \\
\mathbb{B}_{K}^{0, \text { int }} & 0
\end{array}\right)^{-1}\left(\begin{array}{l}
\mathbb{A}_{K}^{\text {int,ext }} \\
\mathbb{B}_{K}^{0, \text { ext }}
\end{array}\right)
$$

and

$$
\widehat{F}_{K}^{\text {ext }}:=F_{K}^{\text {ext }}-\left(\begin{array}{l}
\mathbb{A}_{K}^{\text {int,ext }} \\
\mathbb{B}_{K}^{0, \text { ext }}
\end{array}\right)^{t}\left(\begin{array}{cc}
\mathbb{A}_{K}^{\text {int,int }} & \left(\mathbb{B}_{K}^{0, \text { int }}\right)^{t} \\
\mathbb{B}_{K}^{0, \text { int }} & 0
\end{array}\right)^{-1}\left(\begin{array}{c}
F_{K}^{\text {int }} \\
G_{K}^{0}
\end{array}\right) .
$$

Let us first remark that the element contribution (7.10) has the same unknowns and matrix structure as in the MFD method [17]. Moreover, the matrices $\mathbb{B}_{K}^{1, \text { ext }}$ are identical as in MFDs. Because of the homogeneous Dirichlet boundary condition $(1.1 \mathrm{~b})$, both $F_{K}^{\text {int }}$ and $F_{K}^{\text {ext }}$ are zero; moreover, since we have supposed $g$ constant on $K, G_{K}^{0}=0$ follows. Thus $\widehat{F}_{K}^{\text {ext }}=0$, and we find that also the right-hand sides are the same. It thus remains to check that the matrix $\widehat{\mathbb{A}}_{K}$, or more precisely the scalar product that it generates, satisfies the requirements of stability (3.10) and consistency (5.1) from [17] ( $\widehat{\mathbb{A}}_{K}$ is obviously symmetric). For this purpose, we will rely on the notion of the lifting operator of [17, Theorem 5.1].

Let the notation $\mathbf{V}_{h}(K)$ stand for the restriction of the space $\mathbf{V}_{h}$, defined on the simplicial mesh $\mathcal{T}_{h}$, to the element $K \in \widehat{\mathcal{T}}_{H}$. Let $U_{K}^{\text {ext }}$ be an arbitrary vector associated with the boundary sides $\mathcal{E}_{K}^{\text {ext }}$ of the element $K \in \widehat{\mathcal{T}}_{H}$, representing the exterior normal fluxes through these sides. We will construct a lifting operator which to a given vector $U_{K}^{\text {ext }}$ associates a function $\mathbf{u}_{h, K}$ from $\mathbf{V}_{h}(K)$ such that

$$
\begin{aligned}
\left.\left(\mathbf{u}_{h, K} \cdot \mathbf{n}_{K}\right)\right|_{\sigma} & =\frac{U_{\sigma}^{\text {ext }}}{|\sigma|} \quad \forall \sigma \in \mathcal{E}_{K}^{\mathrm{ext}}, \\
\nabla \cdot \mathbf{u}_{h, K} & =\frac{1}{|K|} \sum_{\sigma \in \mathcal{E}_{K}^{\text {ext }}} U_{\sigma}^{\mathrm{ext}} .
\end{aligned}
$$

This corresponds to the problem (5.4) in [17], with a different scaling (our vector $U_{K}^{\text {ext }}$ represents the normal fluxes). Let us decompose $\mathbf{V}_{h}(K)$ into $\mathbf{V}_{h}(K)^{\text {ext }}$, generated by the basis functions of $\mathbf{V}_{h}(K)$ associated with $\sigma \in \mathcal{E}_{K}^{\text {ext }}$ (oriented along the exterior normal $\mathbf{n}_{K}$ ), and $\mathbf{V}_{h}(K)^{\text {int }}$, generated by the other basis functions of $\mathbf{V}_{h}(K)$. Prescribe $\mathbf{u}_{h, K}^{\text {ext }} \in \mathbf{V}_{h}(K)^{\text {ext }}$ by the fluxes $U_{K}^{\text {ext }}$. Let $\Phi_{h}(K)$ stand for the restriction of the space $\Phi_{h}$, defined on the simplicial mesh $\mathcal{T}_{h}$, to the element $K \in \widehat{\mathcal{T}}_{H}$. Let $\Phi_{h}(K)^{0}$ be its subspace of functions with mean value zero. Then (7.13)-(7.14) can be achieved via the following local Neumann problem: find $\mathbf{u}_{h, K} \in \mathbf{V}_{h}(K), \mathbf{u}_{h, K}=\mathbf{u}_{h, K}^{\text {int }}+\mathbf{u}_{h, K}^{\text {ext }}$, and $p_{h, K}^{0} \in \Phi_{h}(K)^{0}$ such that

$$
\begin{aligned}
\left(\underline{\mathbf{S}}^{-1} \mathbf{u}_{h, K}^{\text {int }}, \mathbf{w}_{h, K}^{\text {int }}\right)_{K}-\left(p_{h, K}^{0}, \nabla \cdot \mathbf{w}_{h, K}^{\text {int }}\right)_{K} & =-\left(\underline{\mathbf{S}}^{-1} \mathbf{u}_{h, K}^{\text {ext }}, \mathbf{w}_{h, K}^{\text {int }}\right)_{K} & & \forall \mathbf{w}_{h, K}^{\text {int }} \in \mathbf{V}_{h}(K)^{\text {int }}, \\
-\left(\nabla \cdot \mathbf{u}_{h, K}^{\text {int }}, \phi_{h, K}^{0}\right)_{K} & =\left(\nabla \cdot \mathbf{u}_{h, K}^{\text {ext }}, \phi_{h, K}^{0}\right)_{K} & & \forall \phi_{h, K}^{0} \in \Phi_{h}(K)^{0} .
\end{aligned}
$$

Whenever $U_{\sigma}^{\text {ext }}$ represents the normal fluxes across the sides of $\mathcal{E}_{K}^{\text {ext }}$ of a constant vector field $\mathbf{u}_{K}$, the solution $\mathbf{u}_{h, K}$ of $(7.15 \mathrm{a})-(7.15 \mathrm{~b})$ obviously coincides with $\mathbf{u}_{K}$, so that the consistency condition (5.5) of [17] is satisfied. Moreover, the stability condition (5.8) of [17] is also classical for the above local Neumann problem. 
It is now crucial to understand that (7.15a)-(7.15b) is nothing but a functional form of (7.7). Indeed, as explained above, $F_{K}^{\text {int }}$ and $G_{K}^{0}$ are both equal to zero in the present setting. Let now the two vectors $U_{K}^{\text {ext }}$ and $V_{K}^{\text {ext }}$ be arbitrary. Let $\mathbf{u}_{h, K}$ be the solution of $(7.15 \mathrm{a})-(7.15 \mathrm{~b})$ for $U_{K}^{\text {ext }}$. Similarly, prescribe $\mathbf{v}_{h, K}^{\text {ext }} \in \mathbf{V}_{h}(K)^{\text {ext }}$ by the fluxes $V_{K}^{\text {ext }}$ and define $\mathbf{v}_{h, K} \in \mathbf{V}_{h}(K)$, $\mathbf{v}_{h, K}=\mathbf{v}_{h, K}^{\text {int }}+\mathbf{v}_{h, K}^{\text {ext }}$, and $q_{h, K}^{0} \in \Phi_{h}(K)^{0}$ by

$$
\begin{aligned}
\left(\underline{\mathbf{S}}^{-1} \mathbf{v}_{h, K}^{\text {int }}, \mathbf{w}_{h, K}^{\text {int }}\right)_{K}-\left(q_{h, K}^{0}, \nabla \cdot \mathbf{w}_{h, K}^{\text {int }}\right)_{K} & =-\left(\underline{\mathbf{S}}^{-1} \mathbf{v}_{h, K}^{\text {ext }}, \mathbf{w}_{h, K}^{\text {int }}\right)_{K} & & \forall \mathbf{w}_{h, K}^{\text {int }} \in \mathbf{V}_{h}(K)^{\text {int }}, \\
-\left(\nabla \cdot \mathbf{v}_{h, K}^{\text {int }}, \phi_{h, K}^{0}\right)_{K} & =\left(\nabla \cdot \mathbf{v}_{h, K}^{\text {ext }}, \phi_{h, K}^{0}\right)_{K} & & \forall \phi_{h, K}^{0} \in \Phi_{h}(K)^{0} .
\end{aligned}
$$

Using the equivalence of (7.15a)-(7.15b) and (7.7), we will now show that there holds

$$
\left(V_{K}^{\text {ext }}\right)^{t} \widehat{\mathbb{A}}_{K} U_{K}^{\text {ext }}=\left(\underline{\mathbf{S}}^{-1} \mathbf{u}_{h, K}, \mathbf{v}_{h, K}\right)_{K}
$$

which is nothing but the definition (5.7) of [17] (for $\underline{\mathbf{S}}$ constant on $K$ ). Thus, all conditions of [17, Theorem 5.1] will be satisfied, proving that the MFE method (7.6) belongs to the MFD family.

In order to show (7.17), remark that the matrix $\widehat{\mathbb{A}}_{K}$ appears in (7.10) obtained upon plugging (7.7) into (7.9). Let $p_{h, K} \in \Phi_{h}(K), p_{h, K}=p_{h, K}^{1}+p_{h, K}^{0}$, with $p_{h, K}^{1} \in \Phi_{h}(K)^{1}$ corresponding to the constant $P_{K}^{1}$ from (7.9). The functional form of (7.9) states that $\mathbf{u}_{h, K}$ and $p_{h, K}$ need to satisfy

$$
\begin{array}{rr}
\left(\underline{\mathbf{S}}^{-1} \mathbf{u}_{h, K}^{\mathrm{ext}}, \mathbf{w}_{h, K}^{\mathrm{ext}}\right)_{K}+\left(\underline{\mathbf{S}}^{-1} \mathbf{u}_{h, K}^{\mathrm{int}}, \mathbf{w}_{h, K}^{\mathrm{ext}}\right)_{K}-\left(p_{h, K}^{1}, \nabla \cdot \mathbf{w}_{h, K}^{\mathrm{ext}}\right)_{K}-\left(p_{h, K}^{0}, \nabla \cdot \mathbf{w}_{h, K}^{\mathrm{ext}}\right)_{K}=0 \\
\forall \mathbf{w}_{h, K}^{\mathrm{ext}} \in \mathbf{V}_{h}(K)^{\mathrm{ext}}, \\
-\left(\nabla \cdot \mathbf{u}_{h, K}^{\mathrm{ext}}, \phi_{h, K}^{1}\right)_{K}=-\left(g, \phi_{h, K}^{1}\right)_{K} & \forall \phi_{h, K}^{1} \in \Phi_{h}(K)^{1},
\end{array}
$$

noting that $\left(\nabla \cdot \mathbf{u}_{h, K}^{\text {int }}, \phi_{h, K}^{1}\right)_{K}=0$ by the Green theorem. Taking $\mathbf{w}_{h, K}^{\text {ext }}=\mathbf{v}_{h, K}^{\text {ext }}$ in (7.18a), we see that

$$
\left(V_{K}^{\text {ext }}\right)^{t} \widehat{\mathbb{A}}_{K} U_{K}^{\text {ext }}=\left(\underline{\mathbf{S}}^{-1} \mathbf{u}_{h, K}^{\text {ext }}, \mathbf{v}_{h, K}^{\text {ext }}\right)_{K}+\left(\underline{\mathbf{S}}^{-1} \mathbf{u}_{h, K}^{\text {int }}, \mathbf{v}_{h, K}^{\text {ext }}\right)_{K}-\left(p_{h, K}^{0}, \nabla \cdot \mathbf{v}_{h, K}^{\text {ext }}\right)_{K},
$$

where $\mathbf{u}_{h, K}^{\text {int }}$ and $p_{h, K}^{0}$ depend on $\mathbf{u}_{h, K}^{\text {ext }}$ through (7.15a)-(7.15b). Now taking $p_{h, K}^{0}$ as test function in $(7.16 \mathrm{~b})$ and $\mathbf{v}_{h, K}^{\text {int }}$ as test function in (7.15a) yields

$$
-\left(p_{h, K}^{0}, \nabla \cdot \mathbf{v}_{h, K}^{\mathrm{ext}}\right)_{K}=\left(\nabla \cdot \mathbf{v}_{h, K}^{\mathrm{int}}, p_{h, K}^{0}\right)_{K}=\left(\underline{\mathbf{S}}^{-1} \mathbf{u}_{h, K}, \mathbf{v}_{h, K}^{\mathrm{int}}\right)_{K} .
$$

Combining the two above relations proves (7.17).

We finally show the second assertion of the theorem. In [27, Theorem 3.1], it was shown that the MFD [17], mixed finite volume [26], and hybrid finite volume methods [31] (or more precisely their generalized forms) on quite general polygonal meshes are all equivalent in the sense that there always exist choices of the parameters of these methods so that they coincide. As we have just demonstrated that MFEs belong to the MFD family, they also belong to this larger family of methods.

We end this section by few remarks:

Remark 7.5 (Comparison with mimetic finite difference, mixed finite volume, and hybrid finite volume methods). The MFE methods on polygonal meshes (7.2) and (7.6) are less restrictive with respect to the mesh $\widehat{\mathcal{T}}_{H}$ than those needed in [17, 26, 31, 27]. The only requirement is the existence of the matching simplicial submesh $\mathcal{T}_{h}$ which needs to be shape-regular for the convergence. Moreover, they admit $\underline{\mathbf{S}}$ and $g$ nonconstant inside the polygonal elements $K \in \widehat{\mathcal{T}}_{H}$ (the alignment is only used with respect to the submesh $\left.\mathcal{T}_{h}\right)$. In our opinion, they can be seen as any of the above methods with optimal choice of the free parameter(s) and improved resolution inside the polygonal cells. 
Remark 7.6 (A priori and a posteriori error estimates). Using Theorems 7.1 and 7.2, (7.2) is only an algebraic rewriting of (7.1), and (7.6) is only an algebraic rewriting of (7.5). Thus, all a priori and a posteriori error estimates known for simplicial meshes are valid for MFE methods also on arbitrary polygonal grids.

Remark 7.7 (Discrete maximum principle). In the same sense as in the previous remark, under the conditions discussed in Section 6, the discrete maximum principle is valid for MFE methods also on arbitrary polygonal grids.

Remark 7.8 (Mixed finite elements with one unknown per polygonal cell). Consider the RT0 case. It turns out that, by the techniques described in Section 4, both (7.2) and (7.6) can, at least in certain situations, be reduced to

$$
\widehat{\mathbb{S}} \widehat{P}=\widehat{H}
$$

i.e., to a system with one unknown per each polygon in $\widehat{\mathcal{T}}_{H}$, cf. [55, Appendix A].

\section{Conclusions}

We have introduced in this paper a systematic way of reducing the number of unknowns in the RT0 MFE method from the flux and potential unknowns to (new) potential unknowns only. This gives rise to a whole family of equivalent one-unknown-per-element reformulations of the MFE method, some of which reduce to well-known discretization schemes. Practically, this can lead to important computational savings. At the same time, various local flux expressions are obtained. We have also recalled the validity of the discrete maximum principle and the possibility to formulate MFE methods on general polygonal meshes.

To us, the major conclusion of the present paper is that in all underlying principles, derivation, properties, applicability, and computational cost, the RT0 MFE method is closely related to many other locally conservative discretization methods. Our main belief is that through these different links, the results and tools available in MFE methods and those available in the context of the previously-cited methods can become mutually available: for MFE methods which possess a well-explored and solid theoretical background, let us cite, e.g., the well-posedness of $(1.3) /(3.2) /(3.3) /(7.1) /(7.5) /(1.7)$ on simplicial meshes, well-posedness of both $(7.2)$ and $(7.6)$ on arbitrary polygonal meshes, the discrete maximum principle in the lowest-order case, optimal convergence and superconvergence a priori error estimates (see, e.g., [16, 47, 23, 24, 54]), optimal a posteriori error estimates $[54,5]$, multigrid methods (see, e.g., $[15,22]$ ), parallel implementations (see, e.g., [35]), multiscale and mortar versions (see, e.g., [9]), and convergence and optimality of adaptive methods [12, 21]; for the previously-cited FV-type methods, let us cite in particular convergence analysis for nonlinear (degenerate) parabolic equations and their systems, see, e.g., $[32,33,30]$.

\section{References}

[1] Aavatsmark, I., Barkve, T., Bøe, Ø., and Mannseth, T. Discretization on unstructured grids for inhomogeneous, anisotropic media. I. Derivation of the methods. SIAM J. Sci. Comput. 19, 5 (1998), 1700-1716.

[2] Agélas, L., Di Pietro, D. A., and Droniou, J. The G method for heterogeneous anisotropic diffusion on general meshes. M2AN Math. Model. Numer. Anal. 44, 4 (2010), $597-625$. 
[3] Agélas, L., and Masson, R. Convergence of the finite volume MPFA O scheme for heterogeneous anisotropic diffusion problems on general meshes. C. R. Math. Acad. Sci. Paris 346, 17-18 (2008), 1007-1012.

[4] Agouzal, A., Baranger, J., Maître, J.-F., and Oudin, F. Connection between finite volume and mixed finite element methods for a diffusion problem with nonconstant coefficients. Application to a convection diffusion problem. East-West J. Numer. Math. 3, 4 (1995), 237254.

[5] Ainsworth, M., and MA, X. Non-uniform order mixed FEM approximation: Implementation, post-processing, computable error bound and adaptivity. J. Comput. Phys. 231, 2 (2012), 436-453.

[6] Alotto, P., And Perugia, I. Mixed finite element methods and tree-cotree implicit condensation. Calcolo 36, 4 (1999), 233-248.

[7] Arbogast, T., And Chen, Z. On the implementation of mixed methods as nonconforming methods for second-order elliptic problems. Math. Comp. 64, 211 (1995), 943-972.

[8] Arbogast, T., Dawson, C. N., Keenan, P. T., Wheeler, M. F., and Yotov, I. Enhanced cell-centered finite differences for elliptic equations on general geometry. SIAM J. Sci. Comput. 19, 2 (1998), 404-425.

[9] Arbogast, T., Pencheva, G., Wheeler, M. F., and Yotov, I. A multiscale mortar mixed finite element method. Multiscale Model. Simul. 6, 1 (2007), 319-346.

[10] Arnold, D. N., And Brezzi, F. Mixed and nonconforming finite element methods: implementation, postprocessing and error estimates. RAIRO Modél. Math. Anal. Numér. 19, 1 (1985), 7-32.

[11] Baranger, J., Maître, J.-F., and Oudin, F. Connection between finite volume and mixed finite element methods. RAIRO Modél. Math. Anal. Numér. 30, 4 (1996), 445-465.

[12] Becker, R., And MaO, S. An optimally convergent adaptive mixed finite element method. Numer. Math. 111, 1 (2008), 35-54.

[13] Beirão da Veiga, L., Lipnikov, K., and Manzini, G. Convergence analysis of the high-order mimetic finite difference method. Numer. Math. 113, 3 (2009), 325-356.

[14] Breil, J., And Maire, P.-H. A cell-centered diffusion scheme on two-dimensional unstructured meshes. J. Comput. Phys. 224, 2 (2007), 785-823.

[15] Brenner, S. C. A multigrid algorithm for the lowest-order Raviart-Thomas mixed triangular finite element method. SIAM J. Numer. Anal. 29, 3 (1992), 647-678.

[16] Brezzi, F., And Fortin, M. Mixed and hybrid finite element methods, vol. 15 of Springer Series in Computational Mathematics. Springer-Verlag, New York, 1991.

[17] Brezzi, F., Lipnikov, K., and Shashkov, M. Convergence of the mimetic finite difference method for diffusion problems on polyhedral meshes. SIAM J. Numer. Anal. 43, 5 (2005), $1872-1896$. 
[18] Cangiani, A., And Manzini, G. Flux reconstruction and solution post-processing in mimetic finite difference methods. Comput. Methods Appl. Mech. Engrg. 197, 9-12 (2008), 933-945.

[19] Cao, Y., Helmig, R., and Wohlmuth, B. I. Geometrical interpretation of the multi-point flux approximation L-method. Internat. J. Numer. Methods Fluids 60, 11 (2009), 1173-1199.

[20] Chavent, G., Younès, A., And Ackerer, P. On the finite volume reformulation of the mixed finite element method for elliptic and parabolic PDE on triangles. Comput. Methods Appl. Mech. Engrg. 192, 5-6 (2003), 655-682.

[21] Chen, L., Holst, M., And Xu, J. Convergence and optimality of adaptive mixed finite element methods. Math. Comp. 78, 265 (2009), 35-53.

[22] Chen, Z. Equivalence between and multigrid algorithms for nonconforming and mixed methods for second-order elliptic problems. East-West J. Numer. Math. 4, 1 (1996), 1-33.

[23] Cockburn, B., And Gopalakrishnan, J. A characterization of hybridized mixed methods for second order elliptic problems. SIAM J. Numer. Anal. 42, 1 (2004), 283-301.

[24] Cockburn, B., and Gopalakrishnan, J. Error analysis of variable degree mixed methods for elliptic problems via hybridization. Math. Comp. 74, 252 (2005), 1653-1677.

[25] Crouzeix, M., and Raviart, P.-A. Conforming and nonconforming finite element methods for solving the stationary Stokes equations. I. Rev. Française Automat. Informat. Recherche Opérationnelle Sér. Rouge 7, R-3 (1973), 33-75.

[26] Droniou, J., And Eymard, R. A mixed finite volume scheme for anisotropic diffusion problems on any grid. Numer. Math. 105, 1 (2006), 35-71.

[27] Droniou, J., Eymard, R., Gallouët, T., And Herbin, R. A unified approach to mimetic finite difference, hybrid finite volume and mixed finite volume methods. Math. Models Methods Appl. Sci. 20, 2 (2010), 265-295.

[28] Edwards, M. G. Unstructured, control-volume distributed, full-tensor finite-volume schemes with flow based grids. Comput. Geosci. 6, 3-4 (2002), 433-452. Locally conservative numerical methods for flow in porous media.

[29] Eymard, R., Gallouët, T., and Herbin, R. Finite volume methods. In Handbook of Numerical Analysis, Vol. VII. North-Holland, Amsterdam, 2000, pp. 713-1020.

[30] Eymard, R., Gallouët, T., and Herbin, R. Cell centred discretisation of non linear elliptic problems on general multidimensional polyhedral grids. J. Numer. Math. 17, 3 (2009), 173-193.

[31] Eymard, R., GallouËt, T., And Herbin, R. Discretization of heterogeneous and anisotropic diffusion problems on general nonconforming meshes SUSHI: a scheme using stabilization and hybrid interfaces. IMA J. Numer. Anal. 30, 4 (2010), 1009-1043.

[32] Eymard, R., Gallouët, T., Herbin, R., and Michel, A. Convergence of a finite volume scheme for nonlinear degenerate parabolic equations. Numer. Math. 92, 1 (2002), 41-82.

[33] Eymard, R., Herbin, R., And Michel, A. Mathematical study of a petroleum-engineering scheme. M2AN Math. Model. Numer. Anal. 37, 6 (2003), 937-972. 
[34] Eymard, R., Hilhorst, D., And Vohralík, M. A combined finite volume-nonconforming/mixed-hybrid finite element scheme for degenerate parabolic problems. Numer. Math. 105, 1 (2006), 73-131.

[35] Glowinski, R., And Wheeler, M. F. Domain decomposition and mixed finite element methods for elliptic problems. In First International Symposium on Domain Decomposition Methods for Partial Differential Equations (Paris, 1987). SIAM, Philadelphia, 1988, pp. 144172.

[36] Hestenes, M. R., And Stiefel, E. Methods of conjugate gradients for solving linear systems. J. Research Nat. Bur. Standards 49 (1952), 409-436 (1953).

[37] Hoffmann, J. Equivalence of the lowest-order Raviart-Thomas mixed finite element method and the multi point flux approximation scheme on triangular grids. Preprint, University of Erlangen-Nürnberg, 2008.

[38] Klausen, R. A., And Russell, T. F. Relationships among some locally conservative discretization methods which handle discontinuous coefficients. Comput. Geosci. 8, 4 (2004), 341-377 (2005).

[39] Kuznetsov, Y., And Repin, S. Convergence analysis and error estimates for mixed finite element method on distorted meshes. J. Numer. Math. 13, 1 (2005), 33-51.

[40] Kuznetsov, Y. A. Mixed finite element method for diffusion equations on polygonal meshes with mixed cells. J. Numer. Math. 14, 4 (2006), 305-315.

[41] Le Potier, C. Schéma volumes finis pour des opérateurs de diffusion fortement anisotropes sur des maillages non structurés. C. R. Math. Acad. Sci. Paris 340, 12 (2005), 921-926.

[42] Lipnikov, K., Shashkov, M., And Yotov, I. Local flux mimetic finite difference methods. Numer. Math. 112, 1 (2009), 115-152.

[43] Maître, J.-F. Calculs exacts pour les éléments finis triangulaires/simpliciaux. Application à une nouvelle présentation de résolutions "à la volumes finis" des formulations mixtes. Talk at "Journée en l'honneur des 60 ans de Jean-Marie Thomas", June 18, 2004, Pau, France, 2004.

[44] Marini, L. D. An inexpensive method for the evaluation of the solution of the lowest order Raviart-Thomas mixed method. SIAM J. Numer. Anal. 22, 3 (1985), 493-496.

[45] NÉdÉleC, J.-C. Mixed finite elements in $\mathbb{R}^{3}$. Numer. Math. 35, 3 (1980), 315-341.

[46] Raviart, P.-A., And Thomas, J.-M. A mixed finite element method for 2nd order elliptic problems. In Mathematical aspects of finite element methods (Proc. Conf., Consiglio Naz. delle Ricerche (C.N.R.), Rome, 1975). Springer, Berlin, 1977, pp. 292-315. Lecture Notes in Math., Vol. 606.

[47] Roberts, J. E., And Thomas, J.-M. Mixed and hybrid methods. In Handbook of Numerical Analysis, Vol. II. North-Holland, Amsterdam, 1991, pp. 523-639.

[48] Russell, T. F., And Wheeler, M. F. Finite element and finite difference methods for continuous flows in porous media. In The Mathematics of Reservoir Simulation. SIAM, Philadelphia, 1983, pp. 35-106. 
[49] SAAD, Y. Iterative methods for sparse linear systems, second ed. Society for Industrial and Applied Mathematics, Philadelphia, PA, 2003.

[50] Sboui, A., Jaffré, J., And Roberts, J. A composite mixed finite element for hexahedral grids. SIAM J. Sci. Comput. 31, 4 (2009), 2623-2645.

[51] Scheichl, R. Decoupling three-dimensional mixed problems using divergence-free finite elements. SIAM J. Sci. Comput. 23, 5 (2002), 1752-1776.

[52] VAN DER Vorst, H. A. Bi-CGSTAB: a fast and smoothly converging variant of Bi-CG for the solution of nonsymmetric linear systems. SIAM J. Sci. Statist. Comput. 13, 2 (1992), 631-644.

[53] Vohralík, M. Equivalence between lowest-order mixed finite element and multi-point finite volume methods on simplicial meshes. M2AN Math. Model. Numer. Anal. 40, 2 (2006), 367391.

[54] Vohralík, M. Unified primal formulation-based a priori and a posteriori error analysis of mixed finite element methods. Math. Comp. 79, 272 (2010), 2001-2032.

[55] Vohralík, M., and Wohlmuth, B. I. From face to element unknowns by local static condensation with application to nonconforming finite elements. Preprint R11024, Laboratoire Jacques-Louis Lions and HAL Preprint 00614633, submitted for publication, 2011.

[56] Wheeler, M. F., And Yotov, I. A multipoint flux mixed finite element method. SIAM J. Numer. Anal. 44, 5 (2006), 2082-2106.

[57] Younés, A., Ackerer, P., And Chavent, G. From mixed finite elements to finite volumes for elliptic PDEs in two and three dimensions. Internat. J. Numer. Methods Engrg. 59, 3 (2004), 365-388.

[58] Younes, A., And Fontaine, V. Hybrid and multi-point formulations of the lowest-order mixed methods for Darcy's flow on triangles. Internat. J. Numer. Methods Fluids 58, 9 (2008), $1041-1062$.

[59] Younés, A., Mose, R., Ackerer, P., And Chavent, G. A new formulation of the mixed finite element method for solving elliptic and parabolic PDE with triangular elements. $J$. Comput. Phys. 149, 1 (1999), 148-167. 